\title{
Planck early results. IV. First assessment of the High Frequency Instrument in-flight performance ${ }^{\star}$
}

Planck HFI Core Team: P. A. R. Ade ${ }^{50}$, N. Aghanim ${ }^{27}$, R. Ansari ${ }^{42}$, M. Arnaud ${ }^{38}$, M. Ashdown ${ }^{36,2}$, J. Aumont $^{27}$, A. J. Banday ${ }^{55,6,44}$, M. Bartelmann ${ }^{54,44}$, J. G. Bartlett ${ }^{1,34}$, E. Battaner ${ }^{57}$, K. Benabed ${ }^{28}$, A. Benoit ${ }^{26}$, J.-P. Bernard ${ }^{55,6}$, M. Bersanelli1 ${ }^{16,21}$, R. Bhatia ${ }^{3}$, J. J. Bock ${ }^{34,7}$, J. R. Bond ${ }^{4}$, J. Borrill ${ }^{43,52}$, F. R. Bouchet ${ }^{28}$, F. Boulanger ${ }^{27}$, T. Bradshaw ${ }^{48}$, E. Bréelle ${ }^{1}$, M. Bucher ${ }^{1}$, P. Camus ${ }^{26}$, J.-F. Cardoso ${ }^{39,1,28}$, A. Catalano ${ }^{1,37}$, A. Challinor ${ }^{32,36,8}$, A. Chamballu ${ }^{24}$, J. Charra ${ }^{27}$, M. Charra ${ }^{27}$, R.-R. Chary ${ }^{25}$, C. Chiang ${ }^{12}$, S. Church $^{53}$, D. L. Clements ${ }^{24}$, S. Colombi $^{28}$, F. Couchot ${ }^{42}$, A. Coulais ${ }^{37}$, C. Cressiot ${ }^{1}$, B. P. Crill ${ }^{34,46}$, M. Crook ${ }^{48}$, P. de Bernardis ${ }^{15}$, J. Delabrouille ${ }^{1}$, J.-M. Delouis ${ }^{28}$, F.-X. Désert ${ }^{23}$, K. Dolag ${ }^{44}$, H. Dole ${ }^{27}$, O. Doré ${ }^{34,7}$, M. Douspis ${ }^{27}$, G. Efstathiou ${ }^{32}$, P. Eng ${ }^{27}$, C. Filliard ${ }^{42}$, O. Forni ${ }^{55,6}$, P. Fosalba ${ }^{29}$, J.-J. Fourmond ${ }^{27}$, K. Ganga ${ }^{1,25}$, M. Giard ${ }^{55,6}$, D. Girard ${ }^{41}$, Y. Giraud-Héraud ${ }^{1}$, R. Gispert ${ }^{27}$, K. M. Górski ${ }^{34,59}$, S. Gratton ${ }^{36,32}$, M. Griffin ${ }^{50}$, G. Guyot ${ }^{22}$, J. Haissinski ${ }^{42}$, D. Harrison ${ }^{32,36}$, G. Helou ${ }^{7}$, S. Henrot-Versilléf2, C. Hernández-Monteagudo ${ }^{44}$, S. R. Hildebrandt ${ }^{7,41,33}$, R. Hills ${ }^{2}$, E. Hivon ${ }^{28}$, M. Hobson ${ }^{2}$, W. A. Holmes ${ }^{34}$, K. M. Huffenberger ${ }^{58}$, A. H. Jaffe ${ }^{24}$, W. C. Jones ${ }^{12}$, J. Kaplan ${ }^{1}$, R. Kneissl ${ }^{17,3}$, L. Knox ${ }^{13}$, G. Lagache ${ }^{27}$, J.-M. Lamarre ${ }^{37}$, P. Lami2 ${ }^{27}$, A. E. Lange ${ }^{25}$, A. Lasenby ${ }^{2,36}$, A. Lavabre ${ }^{42}$, C. R. Lawrence ${ }^{34}$, B. Leriche ${ }^{27}$, C. Leroy ${ }^{27,55,6}$, Y. Longval ${ }^{27}$, J. F. Macías-Pérez ${ }^{41}$, T. Maciaszek ${ }^{5}$, C. J. MacTavish ${ }^{36}$, B. Maffei ${ }^{35}$, N. Mandolesi ${ }^{20}$, R. Mann ${ }^{49}$, B. Mansoux ${ }^{42}$, S. Masi ${ }^{15}$, T. Matsumura ${ }^{7}$, P. McGehee ${ }^{25}$, J.-B. Melin ${ }^{9}$, C. Mercier ${ }^{27}$, M.-A. Miville-Deschênes ${ }^{27,4}$, A. Moneti ${ }^{28}$, L. Montier ${ }^{55,6}$, D. Mortlock ${ }^{24}$, A. Murphy ${ }^{45}$, F. Nati ${ }^{15}$, C. B. Netterfield ${ }^{11}$, H. U. Nørgaard-Nielsen ${ }^{10}$, C. North ${ }^{50}$, F. Noviello ${ }^{27}$, D. Novikov ${ }^{24}$, S. Osborne ${ }^{53}$, C. Paine ${ }^{34}$, F. Pajot ${ }^{27}$, G. Patanchon ${ }^{1}$, T. Peacocke ${ }^{45}$, T. J. Pearson ${ }^{7,25}$, O. Perdereau ${ }^{42}$, L. Perotto ${ }^{41}$, F. Piacentini ${ }^{15}$, M. Piat ${ }^{1}$, S. Plaszczynski ${ }^{42}$, E. Pointecouteau ${ }^{55,6}$, R. Pons ${ }^{55,6}$, N. Ponthieu ${ }^{27}$, G. Prézeau ${ }^{7}, 34$, S. Prunet ${ }^{28}$, J.-L. Puget ${ }^{27}$, W. T. Reach ${ }^{56}$, C. Renault ${ }^{41}$, I. Ristorcelli ${ }^{55,6}$, G. Rocha ${ }^{34,7}$, C. Rosset ${ }^{1}$, G. Roudier ${ }^{1}$, M. Rowan-Robinson ${ }^{24}$, B. Rusholme ${ }^{25}$, D. Santos ${ }^{41}$, G. Savini ${ }^{47}$, B. M. Schaefer ${ }^{54}$, P. Shellard ${ }^{8}$, L. Spencer ${ }^{50}$, J.-L. Starck ${ }^{38,9}$, P. Stassi ${ }^{41}$, V. Stolyarov ${ }^{2}$, R. Stompor ${ }^{1}$, R. Sudiwala ${ }^{50}$, R. Sunyaev ${ }^{44,51}$, J.-F. Sygnet ${ }^{28}$, J. A. Tauber ${ }^{18}$, C. Thum ${ }^{31}$, J.-P. Torre ${ }^{27}$, F. Touze ${ }^{42}$, M. Tristram ${ }^{42}$, F. Van Leeuwen ${ }^{32}$, L. Vibert 27 , D. Vibert ${ }^{40}$, L. A. Wade ${ }^{34}$, B. D. Wandelt ${ }^{28,14}$, S. D. M. White ${ }^{44}$, H. Wiesemeyer ${ }^{30}$, A. Woodcraft ${ }^{50}$, V. Yurchenko ${ }^{45}$, D. Yvon ${ }^{9}$, and A. Zacchei ${ }^{19}$

(Affiliations can be found after the references)

Received 10 January 2011 / Accepted 29 June 2011

\section{ABSTRACT}

The Planck High Frequency Instrument (HFI) is designed to measure the temperature and polarization anisotropies of the cosmic microwave background and Galactic foregrounds in six $\sim 30 \%$ bands centered at 100, 143, 217, 353, 545, and $857 \mathrm{GHz}$ at an angular resolution of 10' $(100 \mathrm{GHz}), 7^{\prime}(143 \mathrm{GHz})$, and 5' (217 GHz and higher). HFI has been operating flawlessly since launch on 14 May 2009, with the bolometers reaching $100 \mathrm{mK}$ the first week of July. The settings of the readout electronics, including bolometer bias currents, that optimize HFI's noise performance on orbit are nearly the same as the ones chosen during ground testing. Observations of Mars, Jupiter, and Saturn have confirmed that the optical beams and the time responses of the detection chains are in good agreement with the predictions of physical optics modeling and pre-launch measurements. The Detectors suffer from a high flux of cosmic rays due to historically low levels of solar activity. As a result of the redundancy of Planck's observation strategy, the removal of a few percent of data contaminated by glitches does not significantly affect the instrumental sensitivity. The cosmic ray flux represents a significant and variable heat load on the sub-Kelvin stage. Temporal variation and the inhomogeneous distribution of the flux results in thermal fluctuations that are a probable source of low frequency noise. The removal of systematic effects in the time ordered data provides a signal with an average noise equivalent power that is $70 \%$ of the goal in the $0.6-2.5 \mathrm{~Hz}$ range. This is slightly higher than was achieved during the pre-launch characterization but better than predicted in the early phases of the project. The improvement over the goal is a result of the low level of instrumental background loading achieved by the optical and thermal design of the HFI.

Key words. instrumentation: detectors - methods: data analysis - instrumentation: photometers - cosmic background radiation cosmology: observations

^ Corresponding author: J.-M. Lamarre, e-mail: jean-michel. lamarre@obspm.fr 


\section{Introduction}

Planck $^{1}$ (Tauber et al. 2010a; Planck Collaboration 2011a) is the third-generation space mission to measure the anisotropy of the cosmic microwave background (CMB). It observes the sky in nine frequency bands covering $30-857 \mathrm{GHz}$ with high sensitivity and angular resolution ranging from $31^{\prime}$ to $5^{\prime}$. The Low Frequency Instrument (LFI; Mandolesi et al. 2010; Bersanelli et al. 2010; Mennella et al. 2011) covers the 30, 44, and $70 \mathrm{GHz}$ bands with amplifiers cooled to $20 \mathrm{~K}$. The High Frequency Instrument (HFI; Lamarre et al. 2010; Planck HFI Core Team 2011a) covers the $100,143,217,353,545$, and $857 \mathrm{GHz}$ bands with bolometers cooled to $0.1 \mathrm{~K}$. Polarization is measured in all but the highest two bands (Leahy et al. 2010; Rosset et al. 2010). A combination of radiative cooling and three mechanical coolers provide the temperatures needed for the detectors and optics (Planck Collaboration 2011b). Two data processing centres (DPCs) calibrate and process the time-ordered data and make maps of the sky (Planck HFI Core Team 2011b; Zacchei et al. 2011). Planck's sensitivity, angular resolution, and frequency coverage make it a powerful instrument for Galactic and extraGalactic astrophysics as well as cosmology. Early astrophysics results are given in Planck Collaboration (2011h-x).

In this paper we describe the in-flight performance of the HFI as measured during the calibration and performance verification (CPV) phase of observations as well as during the scientific survey. It does not attempt to duplicate the content of the Planck pre-launch status papers (Lamarre et al. 2010; Pajot et al. 2010), but rather presents the operational status from an instrumental viewpoint. The instrumental parameters and the data processing reported in the companion paper (Planck HFI Core Team 2011b) together define the properties of the derived scientific products. This paper focuses on the ability of the HFI to measure intensity without any description of its polarization properties, which will be reported in a later publication.

Section 2 summarizes the instrument design. Section 3 focuses on early in-flight operations, the verification phase, and the tuning of the receiver parameters. Section 4 addresses the measurement of the beams on planets and the independent determination of the transfer function and beam shape. The effective beams, which include the effects of the scan strategy and data processing, are presented in Planck HFI Core Team (2011b). Sections 5-7 are dedicated to noise, systematic effects, and instrument stability, respectively. A summary of the HFI inflight performance and a comparison with pre-launch expectations are presented in Sect. 8.

\section{The HFI instrument}

\subsection{Design}

The High Frequency Instrument (HFI) was proposed to ESA in response to the announcement of opportunity for instruments for the Planck mission in 1995. It is designed to measure the sky in six bands (Table 1) with bolometer sensitivities close to the fundamental limit set by photon noise. The lower four frequency bands include channels that are sensitive to linear polarization in addition to total intensity. The capabilities of the HFI are enabled

\footnotetext{
1 Planck (http://wWw.esa.int/Planck) is a project of the European Space Agency (ESA) with instruments provided by two scientific consortia funded by ESA member states (in particular the lead countries France and Italy), with contributions from NASA (USA) and telescope reflectors provided by a collaboration between ESA and a scientific consortium led and funded by Denmark.
}

Table 1. The HFI receivers.

\begin{tabular}{|c|c|c|c|c|}
\hline Band & & $\begin{array}{l}v_{\text {center }} \\
{[\mathrm{GHz}]}\end{array}$ & $\begin{array}{c}\text { Bandwidth } \\
{[\%]}\end{array}$ & $\begin{array}{l}\text { Number of } \\
\text { bolometers }\end{array}$ \\
\hline $100 \mathrm{P}$ & & 100 & 33 & 8 \\
\hline $143 \mathrm{P}$ & $\ldots \ldots \ldots$ & 143 & 32 & 8 \\
\hline 143 & $\ldots \ldots$ & 143 & 30 & 4 \\
\hline $217 \mathrm{P}$ & $\ldots \ldots \ldots \ldots$ & 217 & 29 & 8 \\
\hline 217 & & 217 & 33 & 4 \\
\hline $353 \mathrm{P}$ & & 353 & 29 & 8 \\
\hline 353 & $\ldots \ldots \ldots \ldots$ & 353 & 28 & 4 \\
\hline 545 & $\ldots \ldots \ldots \ldots$ & 545 & 31 & 4 \\
\hline 857 & $\ldots \ldots \ldots \ldots$ & 857 & 30 & 4 \\
\hline
\end{tabular}

Notes. The "P" identifies the polarisation sensitive bolometers.

through a combination of technological advances in each of the critical components needed for bolometric detection:

- Spider web bolometers (SWBs) (Bock et al. 1995; Holmes et al. 2008) and polarization sensitive bolometers (PSBs) (Jones et al. 2003), which can reach the photon noise limit with sufficient bandwidth to enable scanning great circles on the sky at roughly $1 \mathrm{rpm}$. They offer a very low cross-section to cosmic rays that proves to be essential in this environment and with this sensitivity.

- A space qualified 100 mK dilution cooler (Benoît et al. 1997) coupled with a high precision temperature control system.

- The ${ }^{4} \mathrm{He}-\mathrm{JT}$ cooler, an active cooler for $4 \mathrm{~K}$ (Bradshaw \& Orlowska 1997) using vibration controlled mechanical compressors to prevent excessive warming of the $100 \mathrm{mK}$ stage and minimize microphonic effects in the bolometers.

- AC biased readout electronics that provide high sensitivity and low frequency stability (Rieke et al. 1989; Gaertner et al. 1997).

- A thermo-optical design consisting of three corrugated horns and a set of compact reflective filters and lenses at cryogenic temperatures (Church et al. 1996). These include high throughput (multimoded) corrugated horns for the 545 and $857 \mathrm{GHz}$ channels (Murphy et al. 2002).

The angular resolution was chosen to enable the measurement of small scale features in the CMB while providing extremely low levels of stray light contamination. The measurement and removal of Galactic foreground emission requires a large number of bands extending on both sides of the foreground minimum. This is achieved with the six bands of the HFI (Table 1 and Fig. 1) and the three bands of the LFI (Mennella et al. 2011).

The instrument uses a $\sim 20 \mathrm{~K}$ sorption cooler common to the HFI and the LFI (Planck Collaboration 2011b; Bhandari et al. 2000, 2004). The HFI focal plane unit is integrated inside the mechanical structure of the LFI, centered on the focal surface of a common off-axis Gregorian telescope (Tauber et al. 2010a).

The ability to achieve background limited sensitivity was demonstrated by the Archeops balloon-borne experiment (Benoît et al. 2003a,b) - an adaptation of the HFI designed for operation in the environment of a stratospheric balloon. Similarly, the method of polarimetry employed by the HFI was demonstrated by the BOOMERANG experiment (Montroy et al. 2006; Piacentini et al. 2006; Jones et al. 2006). The HFI itself was extensively tested on the ground during the calibration campaigns (Pajot et al. 2010) at IAS in Orsay and CSL at Liège. However, the fully integrated instrument was never characterized in an operational environment like that of the second EarthSun Lagrange point (L2). In addition to thermal and gravitational 


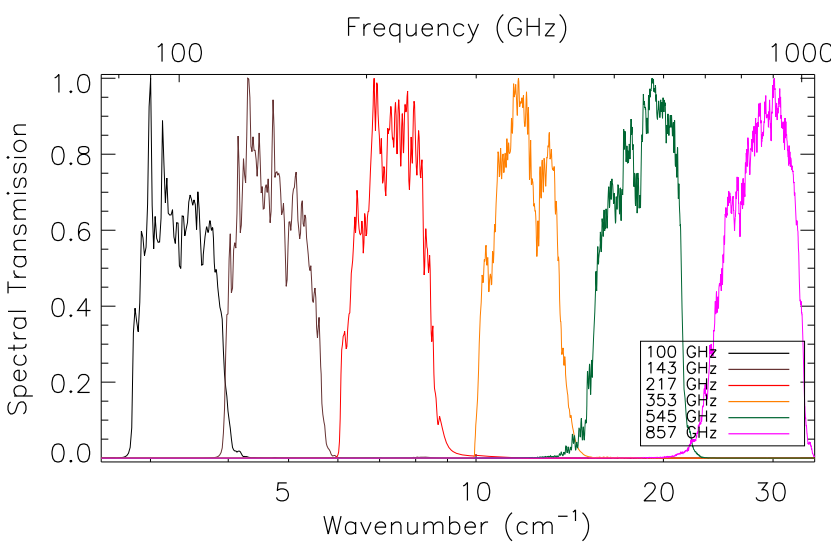

Fig. 1. HFI spectral transmission.

environmental conditions, the spectrum and flux of cosmic rays at L2 is vastly different than that during the pre-flight testing. Finally, due to the operational constraints of the cryogenic receiver, the end to end optical assembly could not be tested on the ground with the focal plane instruments.

The instrument design and development are described in Lamarre et al. (2010). The calibration of the instrument is described in Pajot et al. (2010). The overall thermal and cryogenic design and the Planck payload performance are critical aspects of the mission. Detailed system-level aspects are described in Planck Collaboration (2011a) and Planck Collaboration (2011b).

\subsection{Spectral transmission}

The spectral calibration is described in Pajot et al. (2010) and consists of the end-to-end pre-launch measurements in the vicinity of the passband, combined with component level data to determine the out of band rejection over an extended frequency range (radio-UV). Analysis of the in-flight data shows that the contribution of $\mathrm{CO}$ rotational transitions to the HFI measurements is important. An evaluation of this contribution for the $J=1 \rightarrow 0$ (100 and $143 \mathrm{GHz}$ bands), $J=2 \rightarrow 1(217 \mathrm{GHz}$ band), and $J=3 \rightarrow 2(353 \mathrm{GHz}$ band) transitions of $\mathrm{CO}$ is presented in Planck HFI Core Team (2011b).

\section{Early HFI operation}

\subsection{HFI cool down and cryogenic operating point}

The Planck satellite cooldown is described in Planck Collaboration (2011b). The first two weeks after launch were dedicated to a period of passive outgassing, which ended on 2 June 2009. During this period, gas was circulated through the ${ }^{4} \mathrm{He}-\mathrm{JT}$ cooler and the dilution cooler to prevent clogging by condensable gases. The sorption cooler thermal interface with HFI reached a temperature of $17.2 \mathrm{~K}$ on 13 June. The ${ }^{4} \mathrm{He}-$ JT cooler was only operated at its nominal stroke amplitude of $3.5 \mathrm{~mm}$ on 24 June to leave time for the LFI to carry out a specific calibration with their reference loads around $20 \mathrm{~K}$. On 27 June, the interface with the focal plane unit reached operating temperature of $4.37 \mathrm{~K}$.

The dilution cooler cold head reached 93 mK on 3 July 2009. Taking into account the thermal impact of the LFI tuning, the cool down profile matched, to within a few days, the model derived from the full system cryogenic testing that took place in the summer of 2008 at CSL (Liège).
The regulated operating point of the $4 \mathrm{~K}$ stage was set at $4.8 \mathrm{~K}$ for the $4 \mathrm{~K}$ feed horns on the focal plane unit. The other stages were set to $1.395 \mathrm{~K}$ for the so called $1.4 \mathrm{~K}$ stage, $100.4 \mathrm{mK}$ for the regulated dilution plate, and $103 \mathrm{mK}$ for the regulated bolometer plate.

These numbers, summarised in Table 2, are very close to the planned operational points. As the whole system works nominally, the margins on the interface temperatures and heat lift for the cooling chain are large. The temperature stability of the regulated stages has a direct impact on the scientific performance of the HFI. These stabilities are discussed in detail in Planck Collaboration (2011b) and their impact on the power received by the detectors is given in Sect. 3.3.1. The Planck active cooling chain represents one of the great technological challenges of this mission and has proved to be fully successful.

\subsection{Calibration and performance verification phase}

\subsubsection{Overview}

The calibration and performance verification (CPV) phase of the HFI operations consisted of activities during the initial cooldown to $100 \mathrm{mK}$ and a subsequent period of nominal operation of approximately six weeks before the start of the scientific survey. Activities related to the optimization of the detection chain settings were performed first during the cooldown of the JFET amplifiers, and again during the CPV phase. Most of the operating conditions were pre-determined during the ground calibration; the main uncertainty was the in-flight optical background on the detectors. Other CPV activities included:

- determination of the time response of the detection chain under the in-flight background;

- determination of the channel-to-channel crosstalk;

- characterization of the bolometer response to temperature fluctuations of the $4 \mathrm{~K}$ and $1.4 \mathrm{~K}$ optical stages and to the bolometer plate;

- checking the response of the instrument to the satellite transponder;

- optimization of the numerical compression parameters for the actual sky signal and high energy particle glitch rate;

- measurement of the system response to a range of ring-toring slew amplitudes (1'.7, 2'.0 [nominal], 2'.5);

- measurement of the effect of varying the scan angle with respect to the Sun;

- measurement of the effect of varying the satellite spin rate around the nominal value of $1 \mathrm{rpm}$.

All activities performed during the CPV phase confirmed the pre-launch estimates of the instrument settings and operating mode. We will detail in the following paragraphs the most significant ones.

\subsection{2. ${ }^{4} \mathrm{He}$-JT cooler operation}

The ${ }^{4} \mathrm{He}-\mathrm{JT}$ cooler operating frequency was set to the nominal value of $40.08 \mathrm{~Hz}$ determined during ground tests. Once the cryogenic chain stabilized, the in-flight behaviour of the cooler was similar to that observed during ground tests. A series of narrow lines, resulting from electromagnetic interference from the cooler drive electronics, was observed in the pre-launch testing and is present in the in-flight data. The long term evolution of these "4 K" lines is discussed in Sect. 6.

On 5 August 2009, an unexpected shutdown of the ${ }^{4} \mathrm{He}-\mathrm{JT}$ cooler was triggered by its current regulator. Despite 
Table 2. Main operational and interface parameters of the active cooling chain.

\begin{tabular}{lc}
\hline \hline Interface Sorption cooler- ${ }^{4} \mathrm{He}-J \mathrm{~T}$ cooler (4 K gas pre-cooling temperature) & \\
Interface ${ }^{4} \mathrm{He}-J \mathrm{~J}$ cooler-dilution cooler (dilution gas pre-cooling temperature) & $17.2 \mathrm{~K}$ \\
Interface 1.4 K cooler-dilution gas precooling & $4.37 \mathrm{~K}$ \\
Temperature of dilution plate (after regulation) & $1.34 \mathrm{~K}$ \\
Temperature of bolometer plate (after regulation) & $100.4 \mathrm{mK}$ \\
Temperature of 1.4 K plate (after regulation) & $103 \mathrm{mK}$ \\
Temperature of 4 K plate (after regulation) & $1.395 \mathrm{~K}$ \\
Dilution plate PID power & $4.80 \mathrm{~K}$ \\
Bolometer plate PID power & $24.3-30.7 \mathrm{nW}$ \\
1.4 K PID power & $5.1-7.4 \mathrm{nW}$ \\
4 K PID power & $270 \mu \mathrm{W}$ \\
${ }^{4}$ He-JT cooler stroke amplitude & $1.7 \mathrm{~mW}$ \\
Dilution cooler ${ }^{4} \mathrm{He}$ flow rate & $3450 \mu \mathrm{m}$ \\
Dilution cooler ${ }^{3} \mathrm{He}$ flow rate & $16.19-16.65 \mu \mathrm{mole} / \mathrm{s}$ \\
Present survey life time (started 6 August 2009) & $5.92-6.00 \mu \mathrm{mole} / \mathrm{s}$ \\
\hline
\end{tabular}

investigations into this event, the exact cause remains unexplained. A procedure for a quick restart was developed and implemented in case the problem recurred, but it has not. Six days were required to re-cool the instrument to its nominal operating point.

\subsubsection{Detection chain parameters}

The temperature of the JFET preamplifiers is regulated to minimize the noise. During cooldown, the operational temperature of the JFETs was set while the bolometers themselves were warmer than $130 \mathrm{~K}$ and contributed negligible Johnson noise. The best noise performance of the JFETs is achieved at temperatures near $130 \mathrm{~K}$, in agreement with the ground calibration.

The bolometer bias current and the phase of the synchronous demodulation require in-flight optimization due to the dependence of each on the bolometer impedance, which is itself a function of the optical background.

Figure 2 shows the bolometer response for a set of bias current values measured while Planck was scanning the sky. For this sequence, the satellite spin axis was fixed. For each bias value, the end-to-end noise of the detection chain was computed after subtraction of the sky signal. Ground measurements have shown that the noise equivalent power (NEP) at the maximum response differs from the minimum NEP by less than $1 \%$. Because of its higher signal-to-noise ratio, we use the responsivity to optimize the bias currents (Catalano et al. 2010). The optimum in-flight bias current values correspond to the pre-launch estimates within 5\%. Therefore the pre-launch settings, for which extensive ground characterizations were performed, were kept (Fig. 2). Similarly, the lock-in phase was explored and optimized, and again the pre-launch settings were kept.

The optical background power on the bolometers is at the low end of our conservative range of predictions. This is attributed to a relatively low telescope temperature and no detectable contamination of the telescope surface during launch, resulting in a level of photon noise that is lower than initially expected and a correspondingly improved sensitivity. The telescope temperature and emissivity are discussed in more detail in Planck Collaboration (2011b).

\subsubsection{Data-compression}

The output of the readout electronics unit (REU) consists of one value for each of the 72 science channels for each half-period of modulation (Lamarre et al. 2010). This number, $S_{\mathrm{REU}}$, is the sum of the 40 16-bit ADC signal values obtained within the given half-period. The data processor unit (DPU) performs a lossy quantization of $S_{\text {REU }}$.

We define a compression slice of $254 S_{\text {REU values, corre- }}$ sponding to about $1.4 \mathrm{~s}$ of observation for each detector and to a strip of sky about $8^{\circ}$ long. The mean $\left\langle S_{\mathrm{REU}}\right\rangle$ of the data within each compression slice is computed, and data are demodulated using this mean:

$S_{\mathrm{demod}, i}=\left(S_{\mathrm{REU}, i}-\left\langle S_{\mathrm{REU}}\right\rangle\right) *(-1)^{i}$

where $1<i<254$ is the running index within the compression slice.

The mean $\left\langle S_{\text {demod }}\right\rangle$ of the demodulated data $S_{\text {demod }, i}$ is computed and subtracted, and the resulting data slice is quantized according to a step size $Q$ that is fixed per detector:

$S_{\mathrm{DPU}, i}=\operatorname{round}\left[\left(S_{\mathrm{demod}, i}-\left\langle S_{\mathrm{demod}}\right\rangle\right) / Q\right]$.

This is the lossy part of the algorithm: the required compression factor, obtained through the tuning of the quantization step $Q$, adds a measure of noise to the data. Assuming Gaussian white noise with standard deviation $\sigma$, a quantization setting of $\sigma / Q=$ 2 adds $1 \%$ to the noise (Pajot et al. 2010; Pratt 1978). The value of $\sigma$ was determined at the end of the CPV phase after subtraction of the signal from the timeline.

The two means $\left\langle S_{\text {REU }}\right\rangle$ and $\left\langle S_{\text {demod }}\right\rangle$ are computed as 32-bit words and sent through the telemetry, together with the $S_{\mathrm{DPU}, i}$ values. Variable-length encoding of the $S_{\mathrm{DPU}, i}$ values is performed on board, and the inverse decoding is applied on ground. This provides a lossless transmission of the quantized values.

For a given $Q$ value, the telemetry load from each channel depends on the dynamic range of the signal above the level of the noise. This dynamic range is largest for the high frequency bolometers because of the Galactic signal. The large rate of glitches due to high energy particle interactions contributes significantly to the load from each channel. Optimal use of the bandpass available for the downlink $\left(75 \mathrm{~kb} \mathrm{~s}^{-1} \mathrm{av-}\right.$ erage for HFI science) was obtained initially by using a value of $Q=\sigma / 2.5$ for all bolometer signals, providing a margin with respect to the requirement of $\sigma / Q=2$. The load from each HFI channel is shown and compared to simulated data in Fig. 3. The increase of signal gradients while scanning through the Galactic center in September 2009 triggered the load limitation mechanism (compression error) and up to 80000 samples 
Planck HFI Core Team: Planck early results. IV.
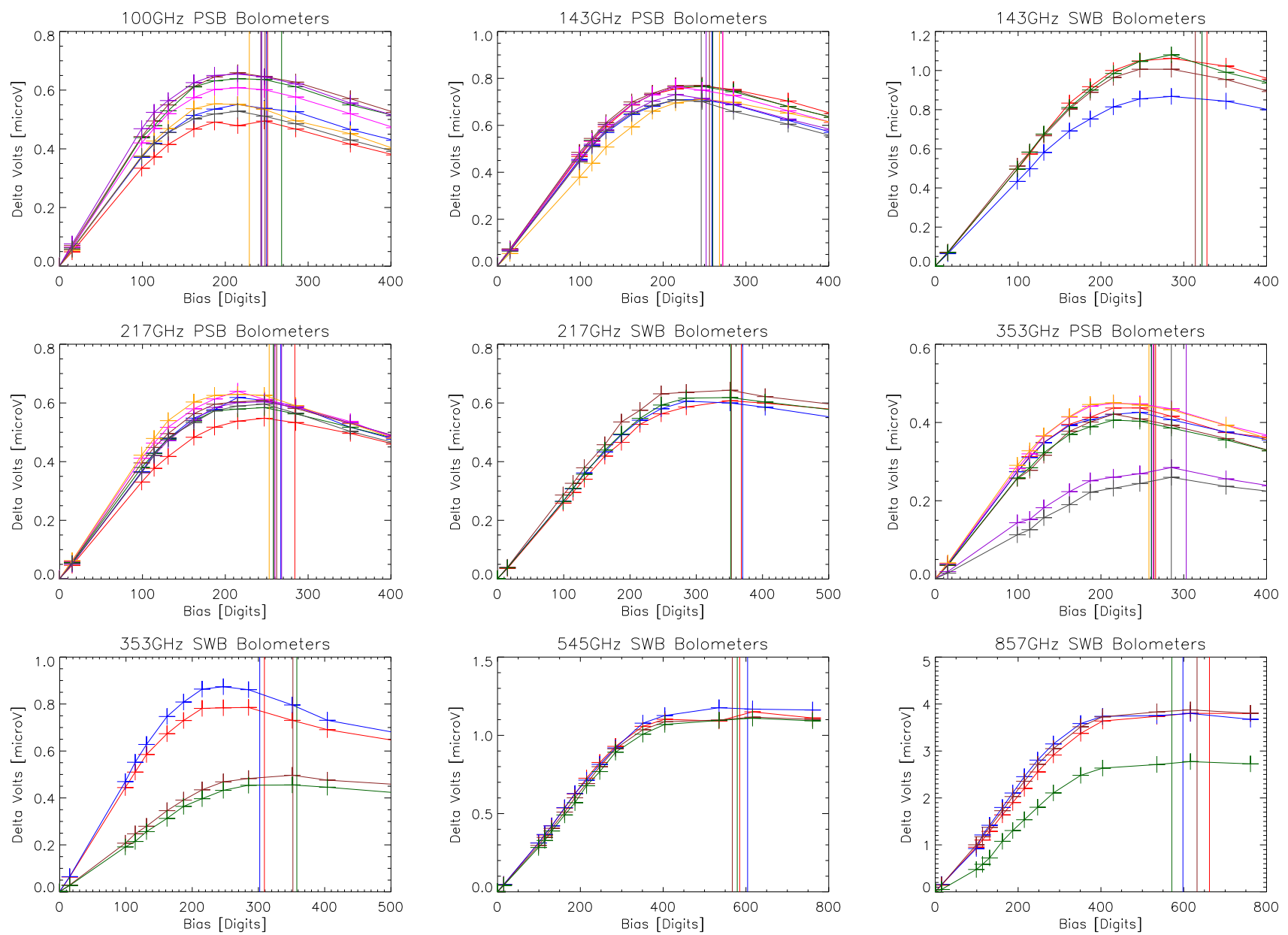

Fig. 2. Optimization of the bolometer bias currents. Vertical lines indicate the final bias value settings. These values are shifted with respect to the maximum because a dynamic response correction (Lamarre et al. 2010) has been taken into account. A bias value of 100 digits corresponds to approximately $0.1 \mathrm{nA}$.

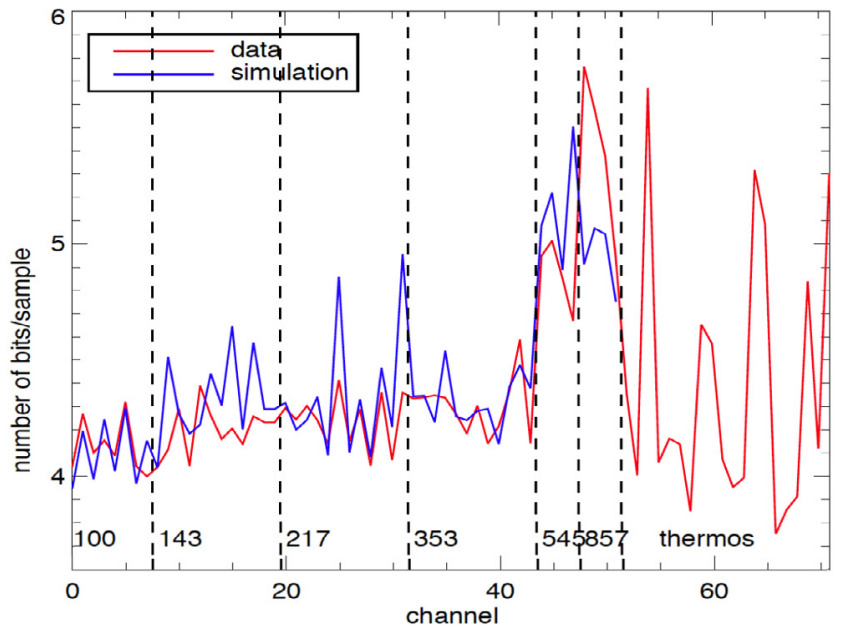

Fig. 3. The telemetry load measured from each HFI channel on 16 July 2010. Simulated data for the same patch of the sky are shown for bolometers. Channels \#54 and higher corrrespond to the precision thermometers on the optical stages of the instruments, plus a fixed resistor (\#60) and a capacitor (\#61) on the bolometer plate.

were lost for each of the $857 \mathrm{GHz}$ band bolometers. Therefore a new value of $Q=\sigma / 2$ was set for those bolometers from 21 December 2009 onward, reducing the number of samples lost

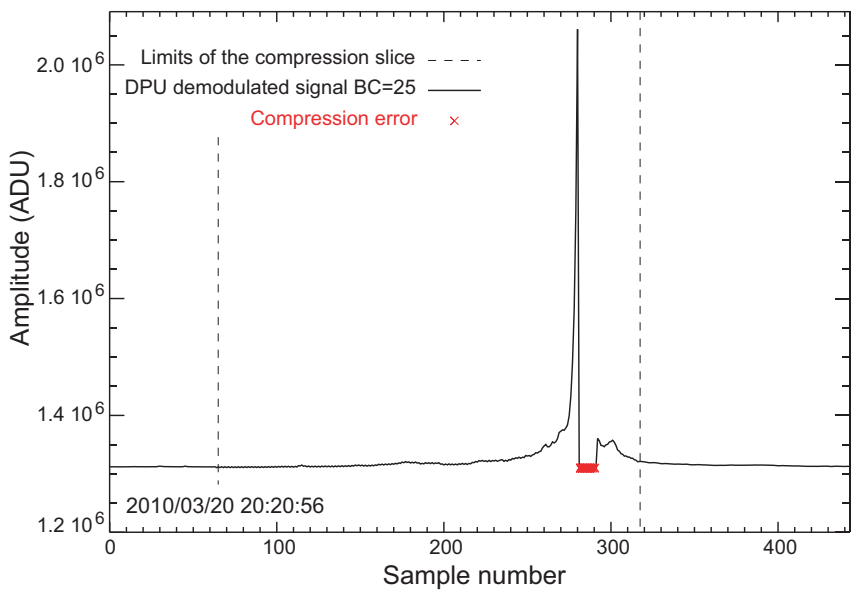

Fig. 4. Example of loss in one compression slice of data on bolometer 857-1. Note the large signal-to-noise ratio while scanning through the Galactic center.

to less than 200 during the following scan through the Galactic center in March 2010. An illustration of a compression error loss is shown in Fig. 4. Due to the redundancy of the Planck scan strategy and the irregular distribution of the few remaining compression errors, no pixels are missing in the maps of the high signal-to-noise ratio Galactic center regions. Periodic checks of 

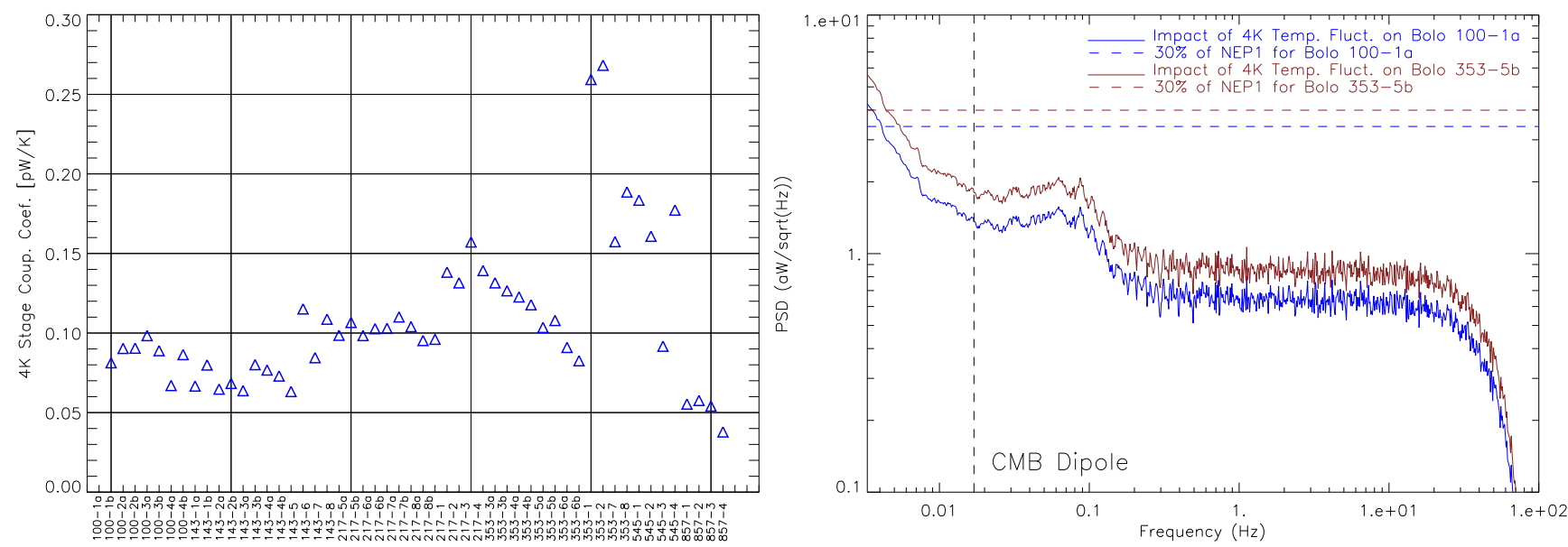

Fig. 5. Left: coupling coefficients of the $4 \mathrm{~K}$ stage. Right: scaled power spectral density (PSD) of the $4 \mathrm{~K}$ stage thermal fluctuations for typical $100 \mathrm{GHz}$ and $353 \mathrm{GHz}$ bolometers (100-1a and 353-5b). The horizontal dashed lines indicate 30\% of the total noise level for these bolometers.
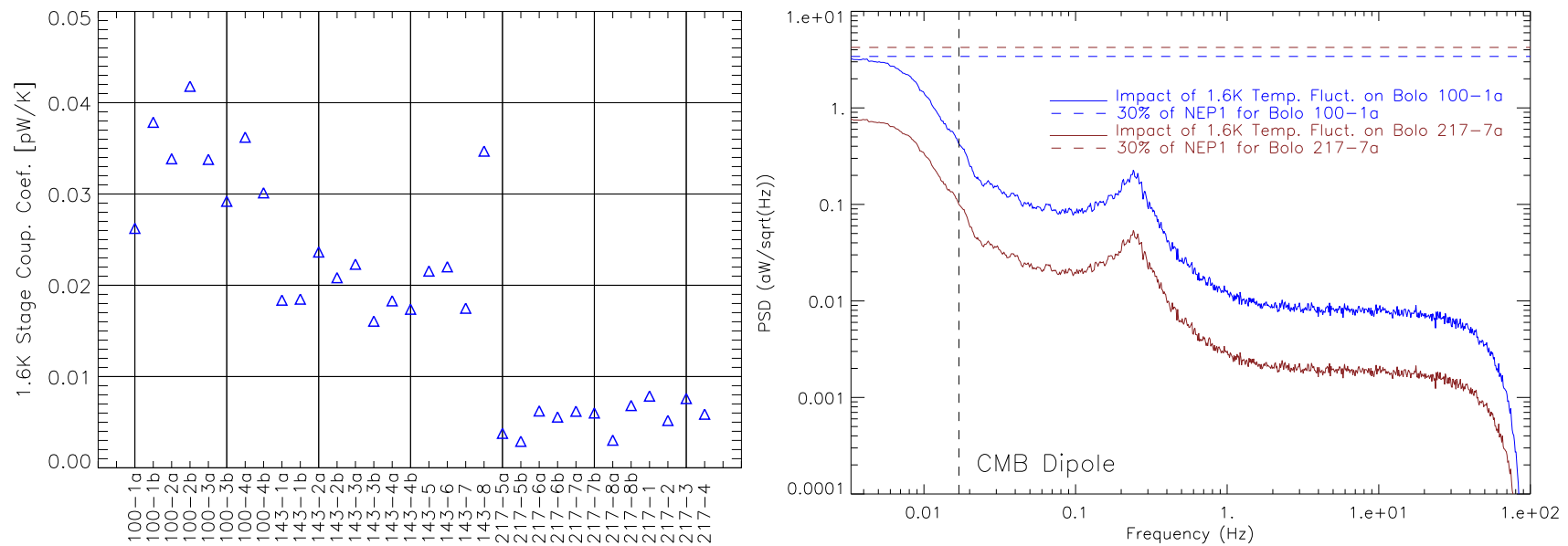

Fig. 6. Left: coupling coefficients of the $1.4 \mathrm{~K}$ stage. The thermal emission in the high frequency bands becomes too small to be measured. Right: scaled PSD of the $1.4 \mathrm{~K}$ stage thermal fluctuations for the 100-1a and 217-7a bolometers. The horizontal dashed lines indicate $30 \%$ of the total noise level of these bolometers.

the noise value $\sigma$ are done for each channel, but no deviation requiring a change in the quantization step $Q$ has been encountered so far.

\subsubsection{First-light survey}

A two-week first-light survey, started on 13 August 2009, allowed an assessment of the quality of the instrument settings, readiness of the data processing chain, and satellite scanning prior to the start of regular science operations. Nominal scientific operations followed, with instrument and satellite parameters unchanged. The data from the first-light survey are included in the definition of the first all-sky survey.

\subsection{Characterization of the HFI}

\subsubsection{Environmental coupling}

The optical background on the HFI bolometers includes contributions from the sky as well as thermal emission of the telescope and from the instrument itself. The operating point of the bolometers is sensitive to the total optical background, and fluctuations in this background have a direct impact on the stability of the HFI measurements.
The in-flight temperature stability of the HFI cryogenic stages is discussed in Planck Collaboration (2011b). The optical coupling of the HFI bolometers to each cryogenic stage is shown in the left panels of Figs. 5 and 6 and in 7. The $100 \mathrm{mK}$ couplings are consistent with pre-launch measurements, showing that no bolometers sustained damage during launch. These couplings are used to estimate the effect of the fluctuations of each cryogenic stage on the bolometer signals. The right panels of Figs. 5 and 6 show the power spectral density (PSD) of the $4 \mathrm{~K}$ and $1.4 \mathrm{~K}$ thermometers, scaled by the optical coupling factors for the most extreme bolometers. The scaled PSDs are compared to the total noise in a typical 100 and $353 \mathrm{GHz}$ channel. The dashed lines correspond to a noise level that is $30 \%$ of the total noise in the signal band of each bolometer. This specification corresponds to a quadratic component smaller than $5 \%$ of the total noise.

\subsubsection{Linearity}

The voltage responsivity of the bolometers to optical power variations is not a strictly linear process; both the conductance between the bolometer and the heat sink and the bolometer impedance have a non-linear dependence on the temperature (see e.g. Catalano 2008; Sudiwala et al. 2000). 
Planck HFI Core Team: Planck early results. IV.

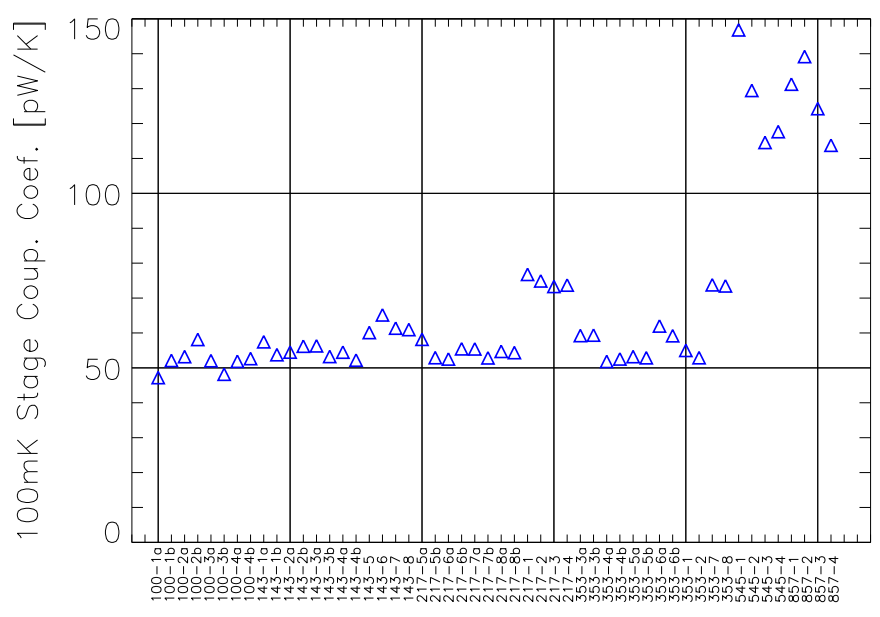

Fig. 7. Bolometer signal coupling coefficients to the $100 \mathrm{mK}$ bolometer plate.

The linearity of the HFI detectors has a direct impact on the calibration of the instrument: a significant change in gain takes place during the Galactic plane crossings and planetary observations, especially in the sub-millimeter bands. The responsivity exhibits a deviation from linearity at the level of a tenth of one percent for Galactic sources (corresponding to a few hundred of attowatts) and of order a few percent for bright sources, such as planets (Pajot et al. 2010). The non-linearity of the response was measured during pre-launch calibrations and during the CPV phase. The responsivity measured in-flight agrees with the ground estimate at a level better than $1 \%$.

The responsivity is modeled to second order in the signal, with the leading term derived from in-flight calibration and the second order derived from pre-launch calibrations. The linearity correction is applied to the timelines using the second order coupling to the signal. Table 3 gives the deviation from linearity on various sources at the center of the beam for the bolometers at each frequency. As indicated in the table, the submillimetre channels saturate the analog to digital converter during a fraction of the modulation period, resulting in a channel dependent non-linearity on these sources that remains uncorrected.

\subsubsection{Electrical crosstalk on HFI detectors}

The electrical coupling of the signal of one bolometer into the readout chain of another, or electrical crosstalk, was measured to be less than $-60 \mathrm{~dB}$ for all pairs of channels during groundbased tests (Pajot et al. 2010). We performed two tests in flight to verify this result, described below.

During the CPV phase we zero the bias on each detector in turn and integrate for ten minutes. The crosstalk coefficient between channels $i$ and $j$ is expressed as:

$C_{i j}=\Delta \tilde{V}_{j} / \Delta \tilde{V}_{i}$,

where $\tilde{V}_{i}$ and $\tilde{V}_{j}$ are the signal voltages in channel $i$ and $j$, corrected for thermal drift. The measured crosstalk matrix, and a histogram of crosstalk levels, are shown in Fig. 8. The crosstalk is mostly confined to nearest neighbours within a belt, where the density of the high impedence signal lines is highest. The measured crosstalk level is in good agreement with ground measurements, typically $<-70 \mathrm{~dB}$, and thus meets the requirement. A few of the polarization sensitive bolometer pairs show crosstalk around $-60 \mathrm{~dB}$.
Table 3. Relative deviation (in \%) from linearity for the CMB dipole, the Galactic center (GC), and planets.

\begin{tabular}{lccccc}
\hline \hline Band & Dipole & GC & Mars & Saturn & Jupiter \\
\hline $100 \mathrm{GHz} \ldots \ldots \ldots$ & $3.8 \times 10^{-4}$ & 0.001 & 0.01 & 0.13 & 0.8 \\
$143 \mathrm{GHz} \ldots \ldots \ldots$ & $1 \times 10^{-3}$ & 0.0017 & 0.02 & 0.18 & 1.0 \\
$217 \mathrm{GHz} \ldots \ldots \ldots$ & $8 \times 10^{-4}$ & 0.003 & 0.05 & 0.53 & 3.2 \\
$353 \mathrm{GHz} \ldots \ldots \ldots$ & $6.4 \times 10^{-4}$ & 0.007 & 0.06 & 0.8 & 4.5 \\
$545 \mathrm{GHz} \ldots \ldots \ldots$ & $<10^{-4}$ & 0.01 & 0.08 & 0.8 & Sat. \\
$857 \mathrm{GHz} \ldots \ldots \ldots$ & $<10^{-4}$ & 0.1 & 0.06 & 0.8 & Sat. \\
\hline
\end{tabular}

Notes. Saturation (Sat.) occurs for the Jupiter measurements at high frequency.

In addition to the electrical test of crosstalk, we measure the channel-to-channel coupling of the cosmic ray impulses and planetary crossings. For completeness, we describe these measurements below; however we feel that the CPV testing and prelaunch calibrations represent a much cleaner measurent of the crosstalk in normal operation.

Thousands of glitch events are collected for one channel, and the signals of all other channels for the same time period are stacked. The voltage crosstalk for individual glitches is defined as

$c_{i j}^{V}=\Delta V_{j} / \Delta V_{i}$

where $V_{i}$ is the glitch amplitude in the channel hit by a cosmic ray, and $V_{j}$ the response amplitude of another channel $j$. Then, for a pair of channels $i$ and $j$, the global voltage crosstalk coefficient is

$C_{i j}^{V}=\operatorname{median}\left(c_{i j}^{V}\right)$.

For SWB channels, in contrast with the CPV results no evidence of crosstalk is seen, with an upper limit of $-100 \mathrm{~dB}$. There are outliers in the submillimetre channels as a result of incomplete flagging of the glitches. A second analysis using the data from planetary crossings instead of glitches gave the same results.

For PSB pairs, we see crosstalk around $-60 \mathrm{~dB}$, in agreement with the CPV tests; however, this is likely an upper limit because it includes the effects of coincident cosmic ray glitches within the pair, which produce a similar signature but are not related to electrical crosstalk.

\section{Beams and time response}

\subsection{Measurement of time response}

\subsubsection{Introduction}

The time response of HFI describes the shift, in amplitude and phase, between the optical signal incident on each detector and the output of the readout electronics. The response can be approximated by a linear complex transfer function in the frequency domain. The signal band of HFI extends from the spin frequency of the spacecraft $\left(f_{\text {spin }} \simeq 16.7 \mathrm{mHz}\right.$ ) to a cutoff defined by the angular size of the beam $(14-70 \mathrm{~Hz}$; see Table 4 from Lamarre et al. 2010). For the channels at 100, 143, 217, and $353 \mathrm{GHz}$, calibration on the dipole normalizes the time response at the spin frequency. To properly measure the small scale signal on the sky, the time response must be characterized to high precision across the entire signal band, spanning four decades from $16.7 \mathrm{mHz}$ to $\sim 100 \mathrm{~Hz}$.

We define the optical beam as the steady state directional response of a given channel to a point source. Any sky signal 


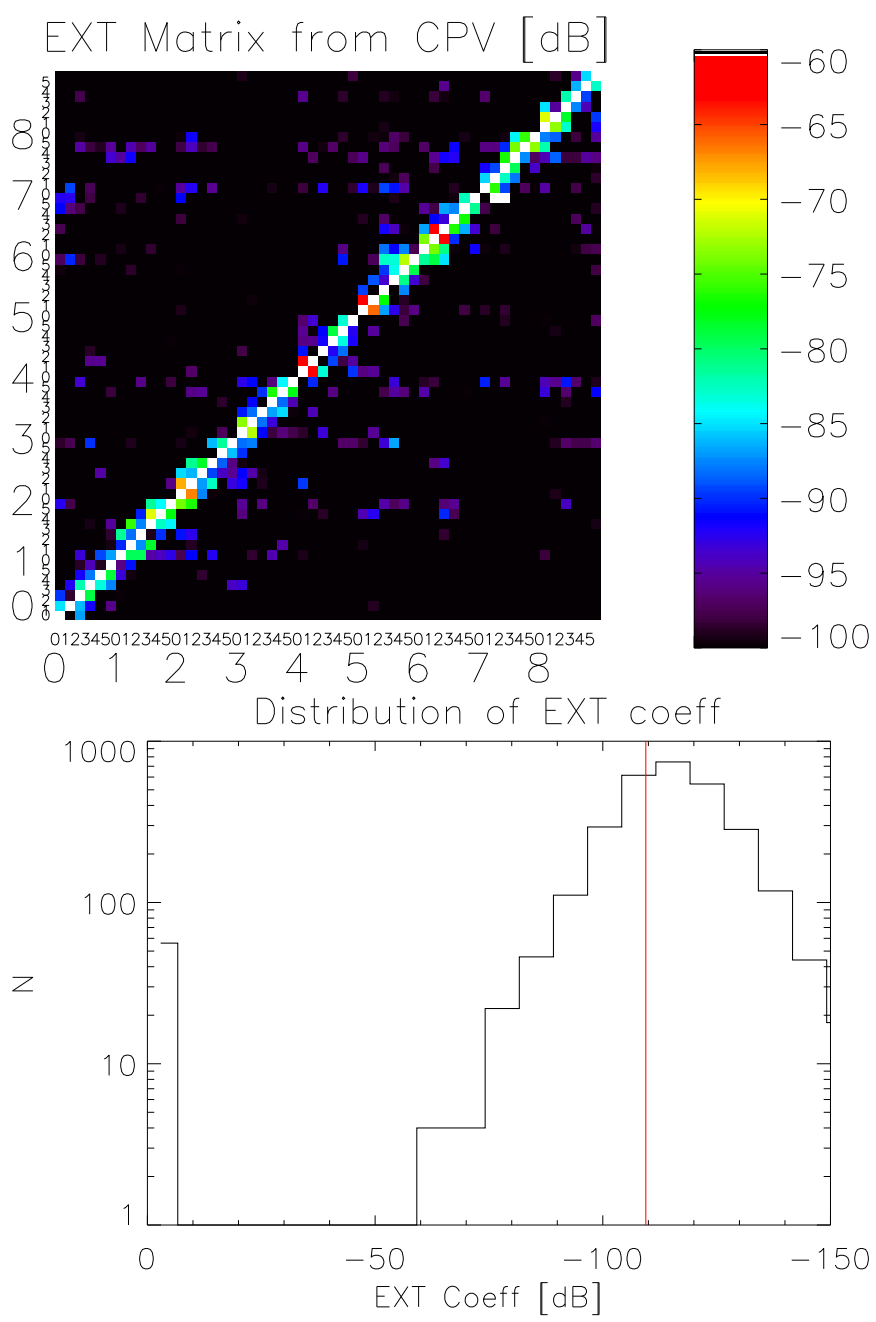

Fig. 8. Top: electrical crosstalk matrix $C_{i j} 54 \times 54$ for all bolometers, coefficients are in dB. Bottom: distribution of electrical crosstalk coefficients in $\mathrm{dB}$.

is convolved with this function, which is completely determined by the optical systems of HFI and Planck.

Since Planck is rotating at a nearly constant rate and around the same direction, the data are the convolution of the signal with both the optical beam and the time response of HFI. We separate the two effects and deconvolve the time response from the time ordered data. This deconvolution results in a flat signal response, but necessarily amplifies any components of the system noise that are not rolled off by the bolometric response. Since it is outside of the signal band, this amplified noise is suppressed by a low-pass filter (Planck HFI Core Team 2011b).

\subsubsection{TF10 model}

The main ingredients of the time response are: (i) heat propagation within the bolometer; (ii) signal modulation at a frequency of $f_{\text {mod }}=90.188 \mathrm{~Hz}$ performed by reversing the bolometer bias current; (iii) the effect of parasitic capacitance along the high impedance wiring between the bolometer and the first electronics stage (JFETs); (iv) band-pass filtering, to reject the low frequency and high frequency white noise in the electronics; (v) signal averaging and sampling; and (vi) demodulation.
Because of the complexity of this sequence, a phenomenological approach is used to model time response. This function is written as the product of three factors:

$H_{10}(f)=H_{\text {bolo }} \times H_{\text {res }} \times H_{\text {filter }}$.

Schematically, the first factor takes into account step (i), the second factor describes a resonance effect that results from the combination of steps (ii), (iii), (v) and (vi), while the purpose of $H_{\text {filter }}$ is to account for step (iv).

Detailed analysis and measurements of heat propagation within the bolometer have shown that $H_{\text {bolo }}$ is given by the algebraic sum of three single pole low pass filters. Explicitly,

$H_{\mathrm{bolo}}=\sum_{i=1,3} \frac{a_{i}}{1+j 2 \pi f \tau_{i}}$,

with 6 parameters $\left(a_{1}, a_{2}, a_{3}, \tau_{1}, \tau_{2}, \tau_{3}\right)$.

$H_{\mathrm{res}}=\frac{1+p_{7}(2 \pi f)^{2}}{1-p_{8}(2 \pi f)^{2}+j p_{9}(2 \pi f)}$,

with 3 free parameters $\left(p_{7}, p_{8}, p_{9}\right)$, and

$H_{\text {filter }}=\frac{1-\left(f / F_{\text {mod }}\right)^{2}}{1-p_{10}(2 \pi f)^{2}+j\left(f / F_{\text {filter }}\right)^{2}}$,

with one free parameter $\left(p_{10}\right)$. A total of 10 free parameters describe this model, as indicated by its name. See Fig. 9 for an illustration of the three components of the time response model TF10 for a typical $217 \mathrm{GHz}$ channel.

The parameter $F_{\text {filter }}$ characterizes the rejection filter width and is fixed to $6 \mathrm{~Hz}$ in the fitting process. Besides the fact that this phenomenological model is physically motivated, this parameterization:

- ensures causality;

- satisfies $H(-f)=H^{*}(f)$;

- asymptotes to 1 when $f$ goes to zero (because we define $a_{1}+a_{2}+a_{3}=1$ ), while it approaches 0 when $f$ goes to infinity; and

- includes enough parameters to provide the necessary; flexibility to fit the measured time response data of all 52 bolometers.

\subsubsection{Fitting the TF10 model to ground data}

To obtain the $10 \times 52$ parameter values, we used three sets of pre-launch measurements. (i) The bolometer response was measured at 10 different frequencies by illuminating all 52 bolometers with a chopped light source. (ii) Other measurements were done using carbon fibers as light sources; the latter were alternately turned on and off at a variable frequency. (iii) The bolometer bias currents were periodically stepped up and lowered by a small amount. By adding a square wave to the DC current, temperature steps are induced, simulating turning on and off a light source (the analysis of these data requires bolometer modelling).

None of these measurements was absolutely normalized; all compared the relative response to inputs of various frequencies. While measurement (i) only provided the amplitude of the time response, measurements (ii) and (iii) provided both the amplitude and the phase. Note that for the phase analysis, because of the lack of precise knowledge of the time origin $t_{0}$ of the light/current pulses, a fourth factor $\exp \left(j 2 \pi \Delta t_{0} f\right)$ is introduced in the expression of $H_{10}(f)$, where the additional parameter $\Delta t_{0}$ represents the uncertainty in time. 


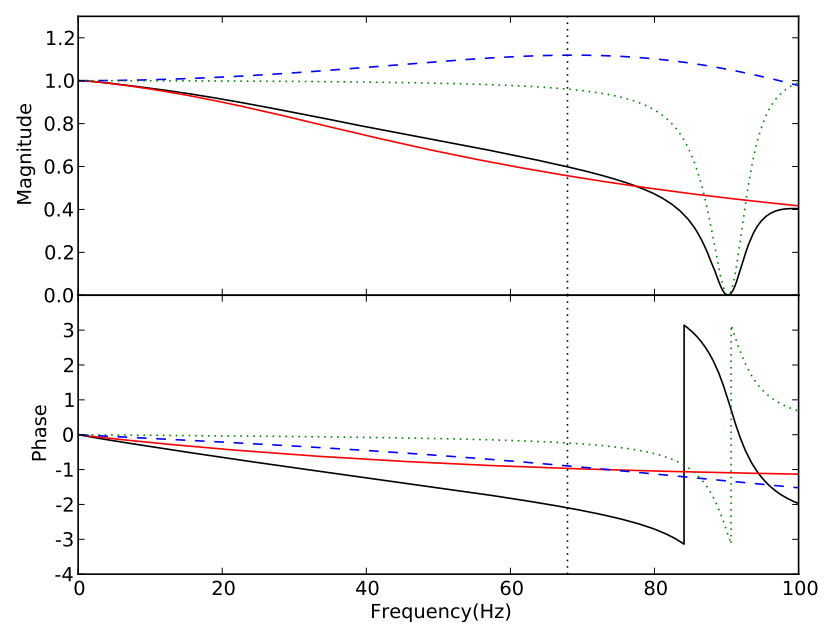

Fig. 9. The amplitude and phase (in radians) of the three components of the TF10 model of the time response. The solid red line is $H_{\text {bolo }}(f)$, the dotted green line shows $H_{\text {filter }}(f)$ and the dashed blue line shows $H_{\text {res }}$. The solid black line is $H_{10}(f)$, the product of the three components. The vertical dotted black line shows the signal frequency where the beam of a $217 \mathrm{GHz}$ channel cuts the signal power by half.

Among the three sets of measurements, the carbon fiber set covered the largest frequency range. Thus it was the best for building the transfer function model described above and for investigating its main features. However, it involves uncertainties that could be resolved only with a very detailed simulation of the set-up, therefore it was not used to calculate the final set of parameter values. The final values were calculated from data sets (i) and (iii), whose frequency ranges are complementary, $2-140 \mathrm{~Hz}$ and $0.0167-10 \mathrm{~Hz}$, respectively. Since no absolute normalization was available, the two datasets were matched in the overlap frequency range.

The fitting of the analytic expression given above to the merged data was done in the range between $16.7 \mathrm{mHz}$ and $120 \mathrm{~Hz}$. The 52 fits have a $\chi^{2} /$ d.o.f. distribution whose mean value is 1.13 , indicating that the model is adequate to describe the data. The numerical values of $p_{10}$ displayed a small spread, $\sigma_{\text {mean }}<6 \times 10^{-4}$. This parameter was set at its mean value to calculate the 52 covariance matrices of the nine remaining parameters, which were useful in propagating the statistical errors.

As described below, the time response thus obtained was further tuned and checked using in-flight observations, in particular signals produced by planets and by cosmic rays (glitches).

\subsubsection{Fitting TF10 to flight data}

The planets Mars, Jupiter, and Saturn are bright, compact sources that are suitable for measuring the beams at 100, 143, 217, and $353 \mathrm{GHz}$. They provide a near-delta-function stimulus to the system that can be used to constrain the time response. Because of the large nonlinear response and highly nonGaussian beams at 545 and $857 \mathrm{GHz}$, we do not perform fits to the planet data at these frequencies, relying instead on prelaunch fits for the time response.

During the first sky survey, Planck observed Mars twice and Jupiter and Saturn once (Planck HFI Core Team 2011b). During a planet observation, the spacecraft scans in its usual observing mode (Planck Collaboration 2011a), shifting the spin axis in $2^{\prime}$ steps along a cycloidal path on the sky. Since planets are close to the ecliptic plane, the coverage in the cross-scan direction is not as fine as in the scan direction. For Jupiter and Saturn, it takes approximately $6 \mathrm{~h}$ (nine periods of stationary pointing, or "rings") for all the bolometers of a given frequency to scan across the planet. Because Mars has a large proper motion, the elapsed time for the first observation lasted $12 \mathrm{~h}$ (or 18 rings).

We use the forward-sense time domain approach (Huffenberger et al. 2010) to simultaneously fit for Gaussian beam parameters and TF10 time response parameters. A custom processing pipeline avoids filtering the data. We extract the raw bolometer signal and demodulate it using the parity bit. We use the flags created by the time ordered information (TOI) processing pipeline to exclude data samples contaminated by cosmic rays, and we additionally flag all data samples where the nonlinear gain correction is more than $0.1 \%$. We use Horizons ${ }^{2}$ ephemerides to compute the pointing of each horn relative to the planet center.

The time domain signal from the planet is modeled as an elliptical Gaussian convolved with the TF10 time response,:

$d(t)=H_{10} \star A(t) G\left[\boldsymbol{x}(t) ; \boldsymbol{x}_{0}, \epsilon, \theta_{\mathrm{FWHM}}, \psi\right]$.

The Gaussian optical beam model $G$ is parameterized as in Eqs. (9)-(11) of Huffenberger et al. (2010). The planet amplitude is parameterized with a disk temperature rather than a single amplitude,

$A(t)=T_{\text {disk }} \frac{\Omega_{\mathrm{p}}(t)}{\Omega_{\mathrm{b}}}$,

where $T_{\text {disk }}$ is the whole-disk temperature of the planet (i.e., the average over the planetary disk), $\Omega_{\mathrm{p}}$ is the solid angle of the planet, which can vary significantly during the observation, and $\Omega_{\mathrm{b}}$ is the solid angle of the beam. $\Omega_{\mathrm{p}}$ is computed using Horizons, which is programmed with Planck's orbit.

The free parameters of the fit are the six parameters of the time response corresponding to $H_{\text {bolo }}$, the two components of the centroid of the beam $\boldsymbol{x}_{0}$, the mean FWHM $\theta_{\text {FWHM }}$, the ellipticity $\epsilon$, the ellipse orientation angle $\psi$, and the planetary disk temperature $T_{\text {disk }}$. The four parameters describing the electronics are somewhat degenerate with the bolometer part of the time response, and we fix them at the ground-based values.

By taking the Fourier transform of the time response function derived on planets, one obtains the system response to a Dirac impulse. This response can be compared to the glitches generated by cosmic rays that deposit energy in the sensor grids.

The glitches detected by HFI are sampled with time steps $1 /\left(2 F_{\text {mod }}\right)$. However, the glitches can be superresolved in time by normalizing, phasing, and stacking single glitch events (Crill et al. 2003). This gives glitch templates for each channel (Alexandre Sauvé, priv. comm.) that are effectively sampled at a much higher frequency.

Figure 10 shows the comparison between a superresolved glitch template and the corresponding calculated response. There is good agreement in general, but there are discrepancies at high frequency ( $f>50 \mathrm{~Hz}$ ). The physical model for the electronics transfer functions briefly described at the end of Sect. 4.1.3 suppresses this discrepancy at high frequency.

Each planetary observation suffers unique systematic effects. Comparison of the time response recovered from multiple observations gives a good assessment of the effects of these systematics. Mars has a large proper motion, giving excellent sampling in the cross-scan direction; however, there is a known diurnal variability in its brightness temperature (Swinyard et al. 2010). Jupiter has a large angular diameter $\left(48^{\prime \prime}\right)$ relative to the

${ }^{2}$ http://ssd.jpl.nasa.gov/?horizons 


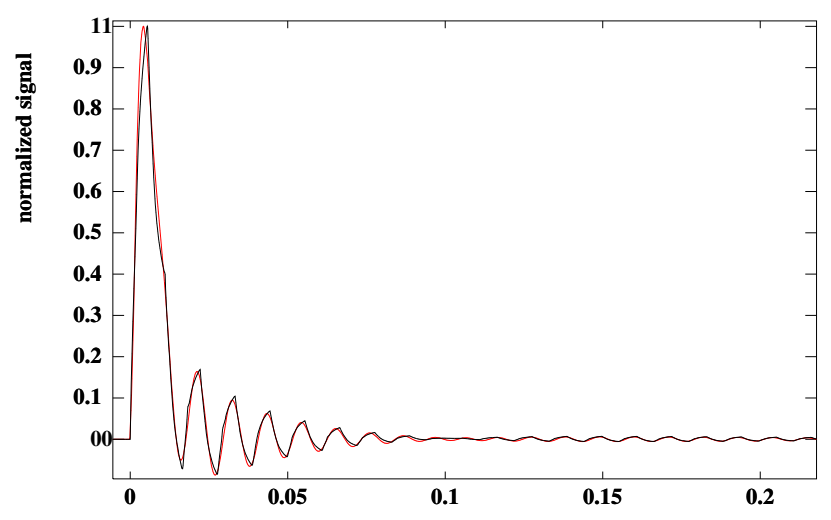

Fig. 10. Comparison of the impulse response of channel 143-2a (red curve) and a template made from stacking glitch events (black curve). Noise begins to dominate further in the timeline. The ringing observed at the modulation frequency is generated by the electronics rejection filter.

HFI beam size, and Saturn and Jupiter are so bright that the HFI detectors are driven significantly nonlinear (see Table 3). Nonetheless, we find that the time response is consistent to $0.1-0.5 \%$ when recovered from each of the planets individually as well as from all planets simultaneously.

A further cross-check is done by stacking planet scans to build a superresolved planet timeline. Time response parameters are fit to the superresolved planet using the assumption of a nearGaussian beam profile, and are consistent with the first approach.

The in-flight time response differs from the ground-based time-response by at worst $1.5 \%$ between $1 \mathrm{~Hz}$ and $40 \mathrm{~Hz}$. We do not include this difference in the final error budget, because it is likely that the time response has changed due to differences in background conditions.

\subsubsection{Low frequency excess response}

The time response of the bolometers is nearly flat from $0 \mathrm{~Hz}$ to a frequency defined by the bolometers' thermal time constant, and then drops sharply at higher frequencies. For the HFI bolometers, the thermal cutoff frequency is $20-50 \mathrm{~Hz}$ (Holmes et al. 2008; Lamarre et al. 2010; Pajot et al. 2010). However, the time response of the HFI exhibits a slight low frequency excess response (LFER) that is typically less than one percent in excess of the response at $1 \mathrm{~Hz}$. We attribute the origin of this effect to the fact that the real thermal properties of the bolometers differ from a simplistic first order model, most likely due to impurities that are weakly connected to the thermometer.

Though the planets are bright, the brief impulse they provide is close to a delta function and the energy is spread evenly across nearly all harmonic components. In combination with low frequency noise, the measurements are not very sensitive to frequencies below $\sim 0.5 \mathrm{~Hz}$; so with planet observations alone we poorly constrain this excess response at very low frequency. Therefore, the ground measurements provide our best estimate of the LFER. In the ground-based measurements the bias step and carbon fiber tests differ by at most $1.5 \%$ at low frequency, so we assign a systematic error of $1.5 \%$ for frequencies below $0.5 \mathrm{~Hz}$.

\subsubsection{Summary of errors in the time response}

As noted above, the data represent the combined effect of the time response and the optical beam. The time response, however, is not degenerate with a Gaussian parameterization of the beam. The true beams deviate from a Gaussian shape at the several percent level near the main lobe, while time response effects tend to give the beam an extended tail following the planet in the scan direction. Although the Gaussian assumption will slightly bias the recovered time response, any residual bias is captured in the measurement of the post-deconvolution scanning beam (Planck HFI Core Team 2011b).

Because of the high signal-to-noise ratio of the planet data, statistical errors in the fit are small, so we assess the systematic errors in the resulting time response by checking the consistency of various methods of recovering the time response. We fit to different combinations of planet data: Mars, Jupiter, and Saturn data separately and all of the data simultaneously to check for systematics resulting from various planets. Additionally we compare the planet-fitted time response with ground-based data and with the impulse response from cosmic ray glitches.

Our final error budget is as follows:

- Low frequency $(f<0.5 \mathrm{~Hz})$ : the errors are dominated by the possibility of a low frequency excess response below $0.5 \mathrm{~Hz}$ at a level of $1.5 \%$ of the total.

- Middle frequency $(0.5 \mathrm{~Hz}<f<50 \mathrm{~Hz})$ : we set an error bar between $0.1 \%$ and $0.5 \%$ depending on the channel. This error bar is set by the consistency in results from different sets of planet data.

- High frequency: $(f>50 \mathrm{~Hz})$ Our empirical model of the electronics in the TF10 model does not describe the system very well at these frequencies, as shown by some disagreement between the glitches and the TF10 impulse response. However, for this data release, the low-pass filter applied to the data and the beam cutoff reduce the importance of this frequency band.

The Planck scan strategy is such that the same region of the sky is observed scanning in nearly opposite directions six months apart. An error in the time response is highlighted in the difference of maps obtained from the first six months and the second six months of the survey. This difference map shows some level of contamination, in particular near the Galactic plane, where the signal amplitude is greatest. The same level of contamination is observed in simulations in which the data are generated with a transfer function, and analysed with a different one, in order to mimic the uncertainties described above. With this technique, we validate the error budget.

\subsection{Optical beams}

The optical beams for each channel are determined by the telescope, the horn antennas in the focal plane and, for the polarized channels, by the orientations of their respective polarization sensitive bolometers (PSBs) (Maffei et al. 2010). Model calculations of the beams are essential, since it was possible to measure only a limited number of beams in the telescope far-field before launch. The 545 and $857 \mathrm{GHz}$ channels, which employ multimoded corrugated horns and waveguides, were not included in this campaign. (The optical beam is related to, but is not the same as the scanning beam defined in Planck HFI Core Team 2011b, and used for data analysis purposes.) Tauber et al. (2010b) reported the best pre-launch expectations for the optical beams, obtained using physical optics calculations with $\mathrm{CORRUG}^{3}$ and GRASP ${ }^{4}$.

\footnotetext{
${ }^{3}$ SMT Consultancies Ltd. http://www. smtconsultancies.co.uk 4 TICRA, http://www.ticra.com
} 
Planck HFI Core Team: Planck early results. IV.

Table 4. Comparison of pre-launch calculations and measured parameters for the HFI optical beams, averaged by frequency band.

\begin{tabular}{lcccccc}
\hline \hline & \multicolumn{3}{c}{ FWHM ['] } & \multicolumn{3}{c}{ ELLIPTICITY } \\
Band & Expected & Mars & $\sigma_{\text {Mars }}$ & Expected & Mars & $\sigma_{\text {Mars }}$ \\
\hline $100 \mathrm{P} \ldots \ldots \ldots$ & 9.58 & 9.37 & 0.06 & 1.17 & 1.18 & 0.006 \\
$143 \mathrm{P} \ldots \ldots \ldots$ & 6.93 & 6.97 & 0.10 & 1.06 & 1.02 & 0.004 \\
$143 \ldots \ldots \ldots$ & 7.11 & 7.24 & 0.10 & 1.03 & 1.04 & 0.005 \\
$217 \mathrm{P} \ldots \ldots \ldots$ & 4.63 & 4.70 & 0.06 & 1.12 & 1.13 & 0.006 \\
$217 \ldots \ldots \ldots \ldots$ & 4.62 & 4.63 & 0.06 & 1.10 & 1.15 & 0.010 \\
$353 \mathrm{P} \ldots \ldots \ldots$ & 4.52 & 4.41 & 0.06 & 1.08 & 1.07 & 0.009 \\
$353 \ldots \ldots \ldots$ & 4.59 & 4.48 & 0.04 & 1.23 & 1.14 & 0.007 \\
$545 \ldots \ldots \ldots$ & 4.09 & 3.80 & $\ldots$ & 1.03 & 1.25 & $\ldots$ \\
$857 \ldots \ldots \ldots$ & 3.93 & 3.67 & $\ldots$ & 1.04 & 1.03 & $\ldots$ \\
\hline
\end{tabular}

Notes. Standard deviations $\sigma$ are computed as the dispersion between the Saturn, Jupiter, and Mars data for each given channel.

Table 4 compares the calculated and measured beams for the single-moded channels. For these channels the pre-launch calculations of FWHM and ellipticity and measured Mars values agree to within a few percent. These differences are contained within $2.7 \sigma$ of the data errors. The main source of discrepancy could be a slight misalignment of the pre-launch telescope model with respect to the actual in-flight telescope geometry, which is currently being investigated (Jensen et al. 2010).

Table 4 also compares pre-launch calculated beams with those measured on Mars. Differences in FWHM are less than $7 \%$. We stress that this discrepancy does not impact the scientific products of the Planck mission since the scanning beams are the ones to be used for data analysis purposes. From an instrumental point of view, the inflight measurements must be considered as the reference for the performance of these channels.

Table 5 reports our best knowledge of the FWHM of the optical beams for each channel. We stress that this table does not provide parameters of the scanning beams, which account for the additional effects of the instrument time response and of the time domain filtering in the data processing (Planck HFI Core Team 2011b).

As reported in Maffei et al. (2010), the $545 \mathrm{GHz}$ and $857 \mathrm{GHz}$ channels are multimoded (more than one electromagnetic mode propagating through the horn antennas) and their optical beams are markedly non-Gaussian. Multimoded horns collect photons from the whole primary mirror but make a beam on the sky large enough to match the scanning strategy and the data rate of the CMB channels at lower frequency. In this way, the highest possible sensitivity is achieved without exceeding the operational limitations of the satellite.

The development of the HFI multimoded channels required the extension of previously existing modelling techniques for the analysis of the corrugated horn antennas and waveguides, as well as for the propagation of partially coherent fields (modes) through the telescope onto the sky (Murphy et al. 2001). Extensive pre-launch measurement campaigns were conducted for all the HFI horn antenna/filter assemblies (Ade et al. 2010). The understanding of these channels through simulations has progressed since Planck was launched, especially in the characterization of their modal content (Murphy et al. 2010).

The similarity of the pre-launch expectations to our current knowledge of the HFI focal plane (beams and their positions on the sky) tells us that the overall structural integrity of the focal plane has been preserved after launch. Furthermore, the optical beams as measured on Mars are shown in Fig. 11 and can be compared with the equivalent representations of the focal plane layout based on calculations in earlier papers (Maffei et al. 2010; Tauber et al. 2010b). A detailed account of the full focal

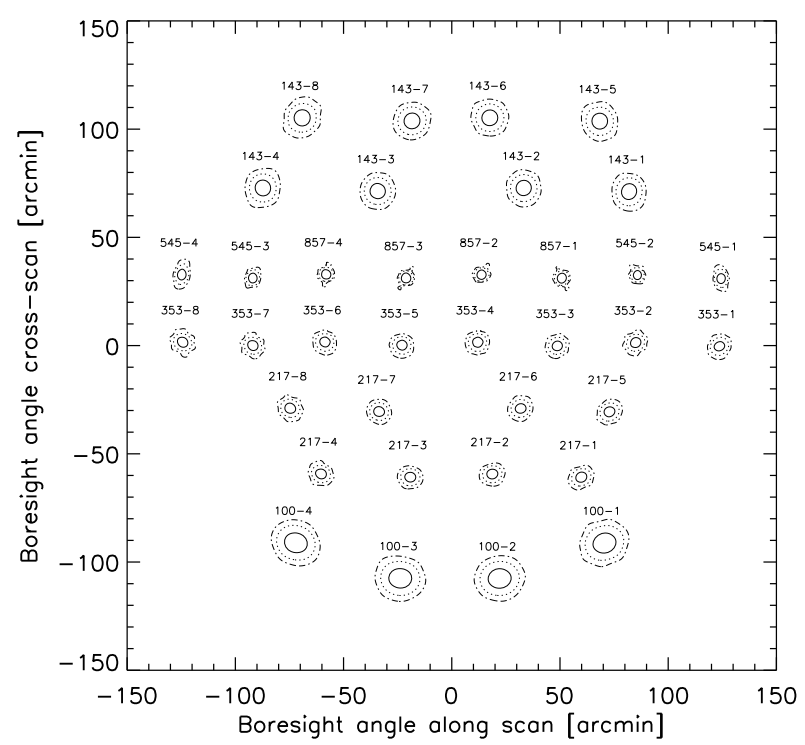

Fig. 11. The distribution of the HFI beams on the sky relative to the telescope boresight as viewed from infinity. Contours of the Gauss-Hermite decomposition of the Mars data at 1\%,10\%, and 50\% power levels from the peak. For the photometers containing a pair of PSBs, the average beam of the two PSBs is shown.

plane reconstruction can be found in Planck HFI Core Team (2011b).

There is a dimpling of the reflector surfaces from the irregular print-through of the honeycomb support structures on the reflector surfaces themselves (Tauber et al. 2010b). GRASP calculations predict that this will generate a series of rings of narrow bright grating lobes around the main lobe. Since the small-scale details of the dimpling structure of the Planck reflectors are irregular, these grating lobes tend to merge with the overall power scattered by the reflector surfaces (Ruze 1966). Figure 12 shows a HEALPIX (Górski et al. 2005) map of the first survey observation of Jupiter minus the second survey observation of the same sky region to remove the sky background. We see the first ring of grating lobes as expected in the map from all 545 and $857 \mathrm{GHz}$ channels, where the signal-to-noise ratio on the planets is highest. The inner $15^{\prime}$ of the beam is saturated and does not appear in the map. At $857 \mathrm{GHz}$, the discrete grating lobes appear at level below $-35 \mathrm{~dB}$ with respect to the peak $(\sim 30 \mathrm{~dB})$, and represent a negligible fraction of the total beam throughput. The shoulder of the beam, extending radially to $\sim 15^{\prime}$, represents a larger 
Table 5. Full width at half maximum (FWHM) of the optical beams and bandpasses for each HFI detector.

\begin{tabular}{|c|c|c|}
\hline Bolometer & $\begin{array}{c}\text { FWHM } \\
{\left[{ }^{\prime}\right]}\end{array}$ & $\begin{array}{c}\text { Frequency range } \\
{[\mathrm{GHz}]}\end{array}$ \\
\hline $100-1 \mathrm{a} \ldots$. & 9.46 & $84.9-113.87$ \\
\hline $100-1 b \ldots$ & 9.60 & $87.0-115.27$ \\
\hline $100-2 \mathrm{a} \ldots$ & 9.41 & $86.5-116.28$ \\
\hline $100-2 b \ldots$ & 9.43 & $84.4-115.42$ \\
\hline $100-3 a \ldots$ & 9.42 & $84.4-116.77$ \\
\hline $100-3 b \ldots$ & 9.47 & $84.4-116.77$ \\
\hline $100-4 a \ldots$ & 9.43 & $84.9-117.79$ \\
\hline $100-4 b \ldots$ & 9.45 & 84.9-117.79 \\
\hline 143-1a .. & 6.91 & $120.8-161.77$ \\
\hline $143-1 b \ldots$ & 6.99 & $120.3-162.78$ \\
\hline $143-2 a \ldots$ & 6.78 & $119.8-162.26$ \\
\hline $143-2 b \ldots$ & 6.80 & $119.3-163.28$ \\
\hline $143-3 a \ldots$ & 6.91 & $120.3-158.73$ \\
\hline $143-3 b \ldots$ & 6.86 & $120.3-160.75$ \\
\hline $143-4 a \ldots \ldots \ldots$ & 7.01 & $118.8-167.83$ \\
\hline $143-4 b \ldots$ & 7.01 & $119.3-161.26$ \\
\hline $143-5 \quad \ldots \ldots \ldots$ & 7.45 & $120.3-166.31$ \\
\hline $143-6$ & 7.08 & $120.3-165.81$ \\
\hline $143-7$ & 7.18 & $120.8-167.83$ \\
\hline $143-8 \quad \ldots \ldots \ldots$ & 7.20 & $120.8-165.3$ \\
\hline $217-5 a \ldots \ldots \ldots$ & 4.73 & $184.0-249.72$ \\
\hline $217-5 b \ldots \ldots \ldots$ & 4.75 & $183.9-249.12$ \\
\hline 217-6a ........ & 4.66 & $182.5-253.26$ \\
\hline $217-6 b \ldots$ & 4.64 & $189.6-252.76$ \\
\hline $217-7 a \ldots$ & 4.63 & $188.6-253.77$ \\
\hline $217-7 b \ldots$ & 4.68 & $189.6-250.74$ \\
\hline $217-8 a \ldots$ & 4.69 & $182.5-253.26$ \\
\hline $217-8 b \ldots \ldots \ldots$ & 4.73 & $182.0-252.76$ \\
\hline $217-1 \quad \ldots \ldots \ldots$ & 4.68 & $189.6-249.72$ \\
\hline $217-2$ & 4.61 & $189.1-253.26$ \\
\hline $217-3$ & 4.59 & $191.1-252.76$ \\
\hline $217-4$ & 4.61 & $193.1-252.76$ \\
\hline $353-3 a \ldots \ldots \ldots$ & 4.47 & $310.9-403.91$ \\
\hline $353-3 b \ldots \ldots \ldots$ & 4.46 & $310.4-405.93$ \\
\hline $353-4 a \ldots \ldots .$. & 4.40 & $323.5-400.88$ \\
\hline $353-4 b \ldots \ldots \ldots$ & 4.39 & $313.9-406.94$ \\
\hline $353-5 a \ldots \ldots .$. & 4.41 & $302.3-405.43$ \\
\hline $353-5 b \ldots \ldots \ldots$ & 4.42 & $299.8-405.93$ \\
\hline 353-6а ........ & 4.47 & $300.3-406.94$ \\
\hline 353-6b ......... & 4.45 & $314.4-397.84$ \\
\hline $353-1 \quad \ldots$ & 4.57 & $310.4-401.38$ \\
\hline $353-2$ & 4.46 & $312.9-407.45$ \\
\hline $353-7$ & 4.44 & $326.1-404.4$ \\
\hline $353-8$ & 4.53 & $318.5-405.92$ \\
\hline$\ldots \ldots \ldots$ & 3.94 & $466.1-638.93$ \\
\hline$\ldots \ldots \ldots$ & 3.63 & $464.5-633.87$ \\
\hline$\ldots \ldots \ldots$ & 3.79 & $467.6-633.87$ \\
\hline $545-4$ & 4.17 & $479.2-635.89$ \\
\hline $857-1$ & 3.73 & 748.1-986.59 \\
\hline $857-2$ & 3.66 & $736.5-982.65$ \\
\hline$\ldots \ldots \ldots$ & 3.76 & $747.1-984.21$ \\
\hline$\ldots \ldots \ldots$ & 3.67 & $744.1-970.02$ \\
\hline
\end{tabular}

Notes. The FWHM is the geometric mean of the major and minor axes of the best fit Gaussian.

contribution to the throughput, ranging from a fraction to several percent of the total solid angle for the CMB and sub-mm channels, respectively.

\section{Noise properties}

The Planck HFI is the first example of space-based bolometers, continuously cooled to $100 \mathrm{mK}$ for several years. Although the

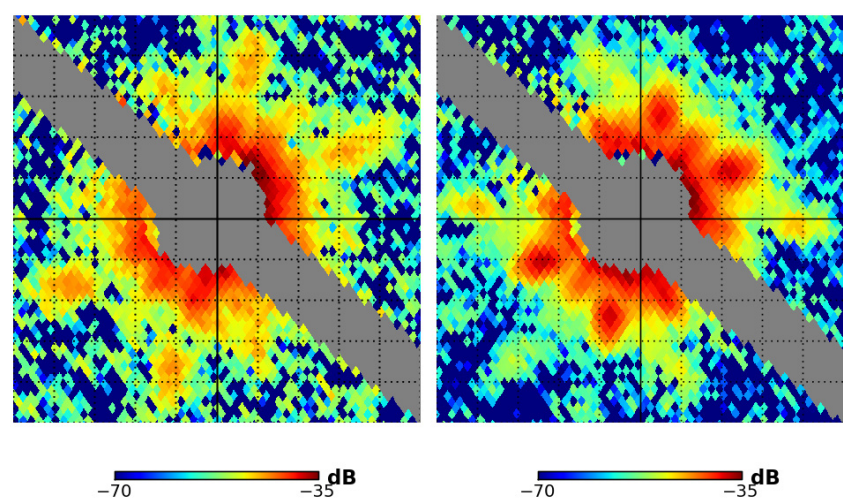

Fig. 12. The "dimpling effect" as seen at $545 \mathrm{GHz}$ (left panel) and $857 \mathrm{GHz}$ (right panel). The grid spacing is $10^{\prime}$. The color scale is in $\mathrm{dB}$ normalized to the peak signal of Jupiter.

detectors were thoroughly tested on the ground (Lamarre et al. 2010; Pajot et al. 2010), it remained to be seen how they would behave in the L2 space environment. We describe here the noise properties of the HFI in the first year of operation, focusing on the differences between space and ground performance.

We start with the Gaussian part of the noise. Section 6 describes the systematic effects that have been analyzed so far.

An example of raw time ordered information (TOI) is shown in Fig. 13. The TOI is dominated by the signal from the CMB dipole, Galactic emission, point sources, and glitches. Therefore, the noise properties cannot be directly deduced from the TOI. We first describe the general method used to evaluate the noise, then we give general statements on the noise properties.

\subsection{Noise estimation}

The Level-2 detnoise pipeline (Planck HFI Core Team 2011b) is used to determine a noise power spectrum, from which one extracts the noise equivalent power (NEP) of the detectors (see Planck HFI Core Team 2011b, for a full description). The pipeline uses redundancies in the observations to determine an estimate of the sky signal, which is then subtracted from the full TOI to produce a pure noise timeline. The signal estimates are the integration of typically 40 circles of data at a constant spin axis pointing. The data are binned in spin phase, providing an accurate estimate of the signal. This signal is then subtracted from the TOI. The residual provides a (slightly biased) estimate of the instantaneous noise. A power spectrum of this residual timeline is derived for each pointing period (see Fig. 14) and fit for the white noise level in the spectral region between 0.6 and $2.5 \mathrm{~Hz}$. The lower limit of $0.6 \mathrm{~Hz}$ is high enough that the low frequency excess noise can be neglected, and the upper limit small enough to keep the time response near to its value at low frequencies $(16 \mathrm{mHz})$ at which the instrument is calibrated.

The noise is stable at a level better than $10 \%$ in the majority of detectors. Exceptions are: (1) a few rings with unusual events that contaminate the measurement, e.g., poorly corrected/flagged glitches or passage over very strong sources such as the Galactic centre, especially at $857 \mathrm{GHz}$; (2) a weak trend, smaller than $1 \%$ in amplitude, that correlates with the duration of the pointing period (an expected bias due to the ring average signal removal); (3) bolometers affected by random telegraph signals (RTS) (see next section); and (4) some uncorrelated jumps in the noise levels for about ten bolometers at the $30 \%$ level for isolated periods of a few days. The overall result is that a very 


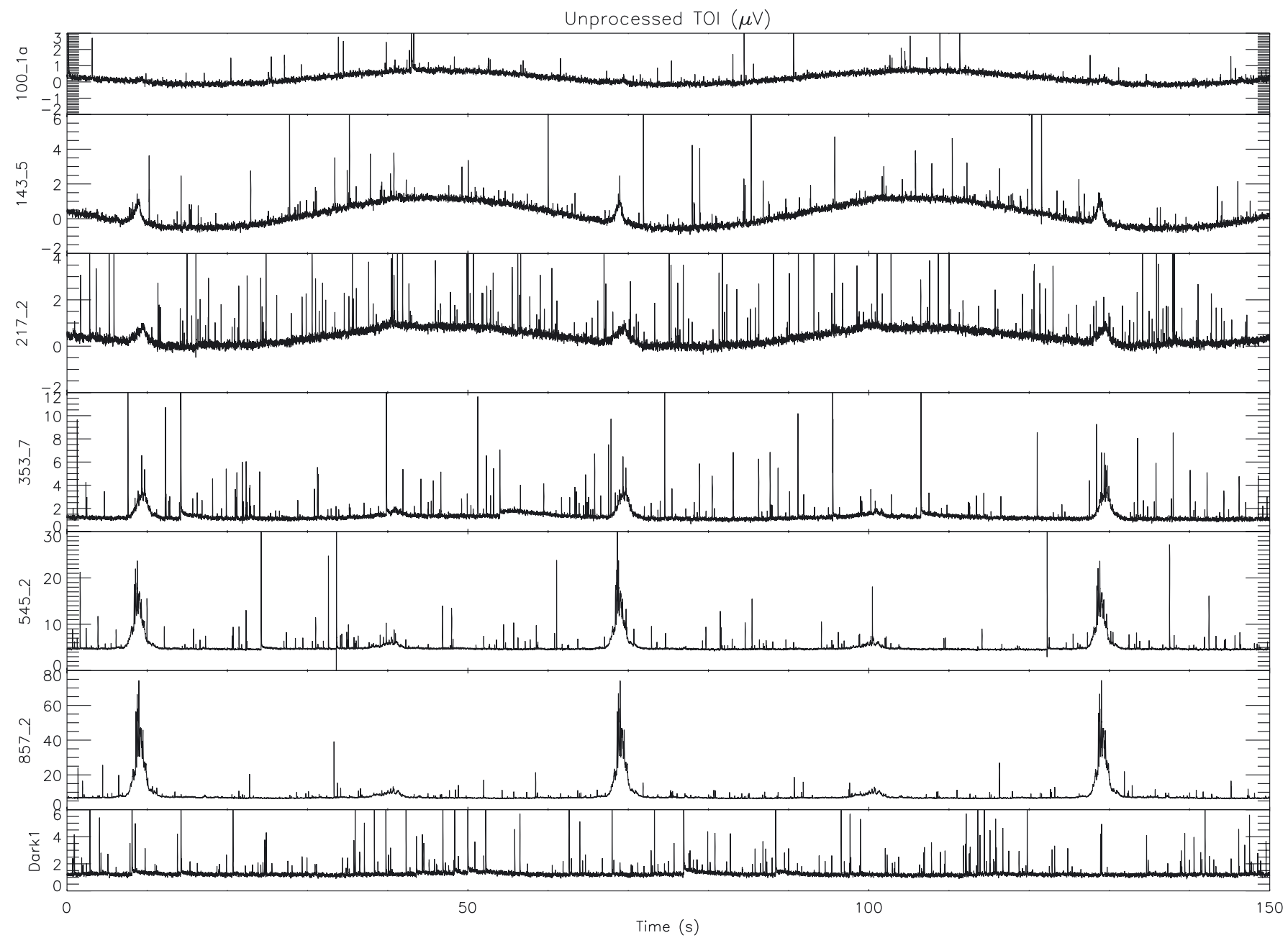

Fig. 13. Examples of raw (unprocessed) TOI for one bolometer at each of six HFI frequencies and one dark bolometer. Slightly more than two scan circles are shown. The TOI is dominated by the CMB dipole, the Galactic dust emission, point sources, and glitches. The relative contribution of glitches is over represented on these plots due to the thickness of the lines that is larger than the real glitch duration.

clear baseline value can be identified and can be used to determine the NEP of each bolometer. This is then converted to the noise equivalent $\Delta T$ (NE $\Delta T)$ with the help of the flux to temperature difference calibration factor. The NE $\Delta T$ s thus measured are given in Table 6 . The quoted uncertainties are derived from the rms of the NEPs in a band around the baseline.

\subsection{The noise components}

The detector noise is described by the combination of several components:

- Photon and bolometer noise, which appear as white noise filtered by the time response of the bolometer, the readout electronics, and the TOI processing.

- Electronics and Johnson noise, which produce noise that is nearly white across the frequency band, but with a sharp decrease at the high frequency end due to the on-board data handling and the TOI filtering.

- The $4 \mathrm{~K}$ lines (Sect 6), appearing as residuals in the spectra.

- The energy deposited by cosmic rays on the bolometers, which appears as "glitches", i.e., positive peaks in the signal, which are removed by the TOI processing (Sect. 6, and
Planck HFI Core Team 2011b). Residuals from glitches appear in the noise spectrum as a bump between 0.1 and $1 \mathrm{~Hz}$. - Low frequency excess noise, which is present below about $100 \mathrm{mHz}$.

The last three sources of noise are detailed in Sect. 6 .

There is an additional noise contribution (of the order of $0.5 \%$ or less) due to the on-board quantization of the data before transmission. In general, the noise level, as measured by the NEP, is between 10 and $20 \mathrm{aW} \mathrm{Hz}^{-1 / 2}$ for the 100 to $353 \mathrm{GHz}$ bands, and between 20 and $40 \mathrm{aW} \mathrm{Hz} z^{-1 / 2}$ for the 545 and $857 \mathrm{GHz}$ bands. These are in line with the ground-based expectations and the lower estimate of the background load, with a detector-to-detector variability of less than $20 \%$ (see Sect. 8).

Due to the AC bias modulation scheme, the $1 / f$ noise from the electronics is aliased near the modulation frequency where it is heavily filtered out. The benefit of this scheme is visible on the noise power spectrum of the $10 \mathrm{M} \Omega$ resistor, which shows a flat spectrum at the Johnson value down to $1 \mathrm{mHz}$, a tribute to the stability of the electronic chain.

At the present time, we assume that the low frequency excess noise, not observed in ground-based measurements, is mostly due to the $100 \mathrm{mK}$ bolometer plate fluctuations. While drifts in the $100 \mathrm{mK}$ stage that are correlated between bolometers are 

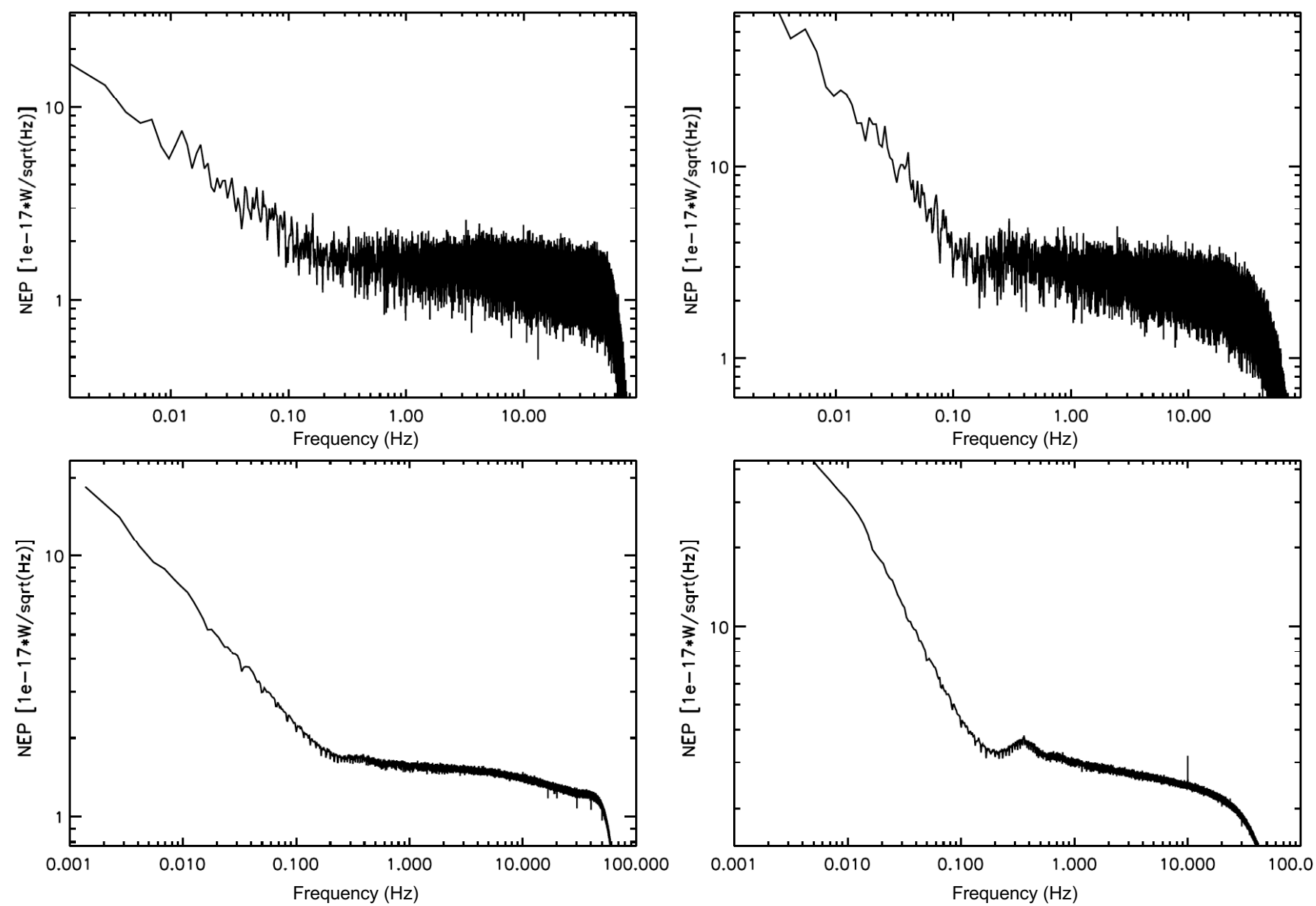

Fig. 14. Typical power spectrum amplitude of bolometers 143-5 and 545-2. For the upper panels, this is the power spectrum density of valid samples, after an average ring (the sky signal) has been subtracted from the TOI. Stacking of the result for 200 rings is shown in the lower panel. Here, the instrument time response is not deconvolved from the data.

removed, there are likely local temperature fluctuations due to the anisotropic distribution of particle energy across the bolometer plate.

\section{First assessment of systematic effects}

\subsection{K lines}

The fundamental frequency of the ${ }^{4} \mathrm{He}-\mathrm{JT}$ cooler $(40.083373 \mathrm{~Hz})$ is phase-locked to the frequency of the data acquisition $(180.37518 \mathrm{~Hz})$ in a ratio of 2 to 9. EMI/EMC impacts the TOI only as very narrow lines. Unfortunately, unlike in ground-based measurements, these lines are not stable in flight. The $4 \mathrm{~K}$ line variations are illustrated in Fig. 15. The variability of the lines is in part due to temperature fluctuations in the service module of the Planck spacecraft. Indeed, some of the variability was related to the power cycling of the data transponder which, for stability reasons, has been kept on continuously since 25 January 2010 (OD 258, Planck Collaboration 2011b, see Fig. 16).

\subsection{Anomolous noise}

Of the 54 bolometers, three - 143-8, 545-3, and 857-4 - show significant random telegraphic signal (RTS), also known as "popcorn noise", Fig. 17 illustrates their behaviour. The noise

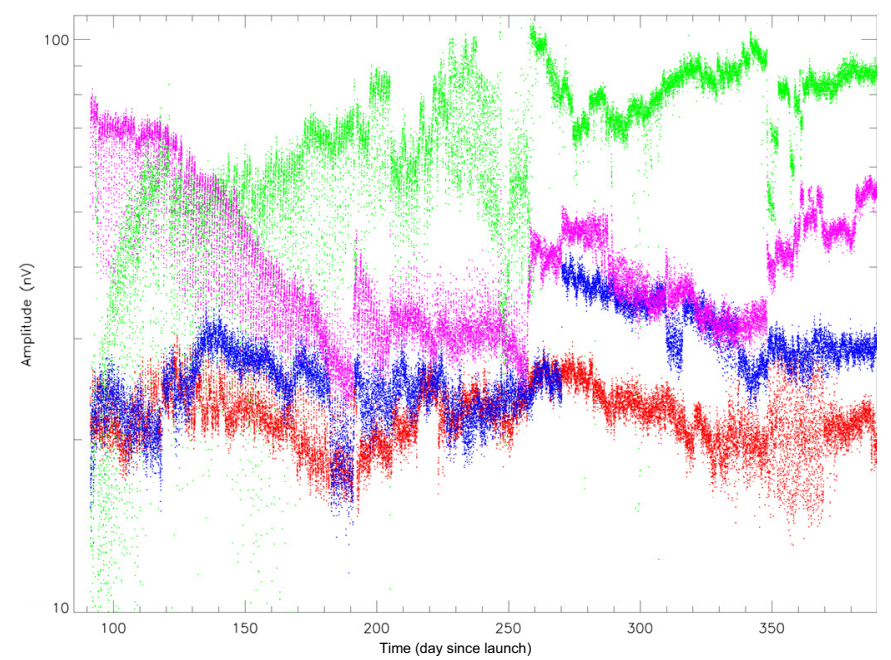

Fig. 15. Typical trend of the cosine and sine coefficient variation of the four main $4 \mathrm{~K}$ lines measured in the TOI processing on the test resistance at $10,30,50$, and $70 \mathrm{~Hz}$.

time line clearly exhibits a few preferred levels. The three bolometers affected by RTS in flight are the same ones for which it occurred most frequently in ground measurements. However, the RTS is variable over time: 1) the level differences can take 
Planck HFI Core Team: Planck early results. IV.

Table 6. $\mathrm{NEP}_{1}$ is the average NEP of the processed signal in the band $0.6-2.5 \mathrm{~Hz}$. $\mathrm{NEP}_{2}$ is the white noise component of the NEP (see text).

\begin{tabular}{|c|c|c|c|c|c|c|c|}
\hline \multirow[b]{2}{*}{ Bolometer } & \multirow[b]{2}{*}{ \# } & \multicolumn{2}{|c|}{ NOISE } & \multicolumn{2}{|c|}{$\mathrm{NEP}_{2}$ FIT } & \multicolumn{2}{|c|}{$\mathrm{NE} \Delta T$} \\
\hline & & $\begin{array}{l}\mathrm{NEP}_{1} \\
{\left[10^{-17}\right.}\end{array}$ & $\begin{array}{r}\mathrm{NEP}_{2} \\
\left.\mathrm{WHz}^{-1}\right]\end{array}$ & $\begin{array}{c}f_{\text {knee }} \\
{[\mathrm{mHz}]}\end{array}$ & $\alpha$ & $\begin{array}{c}\mathrm{CMB} \\
{\left[\mu \mathrm{K}_{\mathrm{CMB}} \mathrm{s}^{1 / 2}\right]}\end{array}$ & $\begin{array}{c}\mathrm{RJ} \\
{\left[\mu \mathrm{K}_{\mathrm{RJ}} \mathrm{S}^{1 / 2}\right]}\end{array}$ \\
\hline $100-1 \mathrm{a} \ldots . . . . .$. & 1 & 1.13 & 1.04 & 218 & 0.93 & 78 & 60.7 \\
\hline $100-1 b \ldots \ldots \ldots$ & 2 & 1.21 & 1.14 & 166 & 1.02 & 69 & 53.0 \\
\hline $100-2 \mathrm{a} .$. & 3 & 1.22 & 1.16 & 126 & 0.96 & 56 & 42.9 \\
\hline $100-2 b$. & 4 & 1.31 & 1.22 & 182 & 0.95 & 58 & 44.7 \\
\hline $100-3 a \ldots$ & 5 & 1.22 & 1.16 & 117 & 1.01 & 61 & 47.4 \\
\hline $100-3 b \ldots$ & 6 & 1.09 & 1.01 & 173 & 1.02 & 66 & 51.4 \\
\hline $100-4 a \ldots$ & 7 & 1.23 & 1.18 & 109 & 0.97 & 59 & 45.2 \\
\hline $100-4 b \ldots$ & 8 & 1.18 & 1.08 & 212 & 0.95 & 70 & 53.6 \\
\hline $143-1 \mathrm{a} \ldots \ldots \ldots$ & 9 & 1.35 & 1.31 & 91 & 1.11 & 50 & 30.4 \\
\hline $143-1 b \ldots \ldots \ldots$ & 10 & 1.18 & 1.09 & 197 & 1.01 & 51 & 30.6 \\
\hline $143-2 a \ldots$ & 11 & 1.28 & 1.20 & 161 & 0.97 & 50 & 30.3 \\
\hline $143-2 b \ldots$ & 12 & 1.30 & 1.27 & 106 & 1.18 & 50 & 30.1 \\
\hline $143-3 a$. & 13 & 1.35 & 1.26 & 202 & 1.01 & 53 & 32.2 \\
\hline $143-3 b$. & 14 & 1.18 & 1.09 & 190 & 1.02 & 51 & 30.9 \\
\hline $143-4 a \ldots$ & 15 & 1.27 & 1.18 & 185 & 0.99 & 53 & 31.7 \\
\hline $143-4 b \ldots$ & 16 & 1.32 & 1.24 & 161 & 1.07 & 59 & 35.5 \\
\hline $143-5 \ldots$ & 17 & 1.53 & 1.46 & 138 & 1.10 & 40 & 23.9 \\
\hline $143-6$ & 18 & 1.37 & 1.25 & 230 & 1.03 & 40 & 24.1 \\
\hline $143-7$. & 19 & 1.49 & 1.40 & 154 & 1.09 & 40 & 23.8 \\
\hline $143-8^{a} \ldots$ & 20 & 2.2 & 1.60 & 1244 & 0.90 & 55 & 33.1 \\
\hline $217-5 a \ldots \ldots \ldots$ & 21 & 1.35 & 1.30 & 117 & 1.10 & 82 & 26.4 \\
\hline $217-5 b \ldots \ldots \ldots$ & 22 & 1.33 & 1.22 & 219 & 1.06 & 81 & 25.9 \\
\hline 217-6а ........ & 23 & 1.30 & 1.25 & 107 & 1.07 & 78 & 25.1 \\
\hline 217-6b ......... & 24 & 1.31 & 1.26 & 118 & 1.08 & 79 & 25.2 \\
\hline $217-7 a \ldots \ldots \ldots$ & 25 & 1.41 & 1.36 & 98 & 1.07 & 80 & 25.4 \\
\hline $217-7 b \ldots \ldots \ldots$ & 26 & 1.25 & 1.17 & 157 & 1.05 & 73 & 23.4 \\
\hline $217-8 \mathrm{a} \ldots \ldots \ldots$ & 27 & 1.37 & 1.31 & 148 & 1.05 & 80 & 25.5 \\
\hline $217-8 b \ldots$ & 28 & 1.27 & 1.17 & 206 & 1.03 & 78 & 24.9 \\
\hline 217-1 . & 29 & 1.59 & 1.49 & 187 & 1.14 & 66 & 20.7 \\
\hline $217-2$ & 30 & 1.61 & 1.48 & 229 & 1.10 & 69 & 21.7 \\
\hline $217-3$ & 31 & 1.63 & 1.54 & 165 & 1.12 & 66 & 20.8 \\
\hline $217-4$ & 32 & 1.62 & 1.53 & 173 & 1.14 & 68 & 21.3 \\
\hline $353-3 a \ldots \ldots \ldots$ & 33 & 1.53 & 1.43 & 174 & 0.98 & 305 & 21.9 \\
\hline $353-3 b \ldots \ldots \ldots$ & 34 & 1.39 & 1.31 & 162 & 1.06 & 282 & 20.3 \\
\hline $353-4 a \ldots \ldots \ldots$ & 35 & 1.34 & 1.28 & 124 & 1.04 & 324 & 22.6 \\
\hline $353-4 b \ldots \ldots \ldots$ & 36 & 1.30 & 1.25 & 127 & 1.12 & 313 & 21.8 \\
\hline $353-5 a \ldots \ldots \ldots$ & 37 & 1.26 & 1.21 & 121 & 1.05 & 268 & 19.4 \\
\hline $353-5 b \ldots \ldots \ldots$ & 38 & 1.33 & 1.27 & 125 & 1.09 & 281 & 20.3 \\
\hline 353-6a ........ & 39 & 1.47 & 1.38 & 208 & 1.08 & 429 & 30.7 \\
\hline 353-6b ......... & 40 & 1.33 & 1.26 & 179 & 1.20 & 432 & 32.4 \\
\hline $353-1 \quad \ldots \ldots \ldots$ & 41 & 1.59 & 1.52 & 100 & 1.04 & 192 & 13.7 \\
\hline $353-2$ & 42 & 1.72 & 1.66 & 98 & 1.07 & 189 & 13.4 \\
\hline $353-7$ & 43 & 1.62 & 1.54 & 155 & 1.18 & 237 & 16.4 \\
\hline $353-8$ & 44 & 1.67 & 1.59 & 159 & 1.15 & 260 & 17.6 \\
\hline $545-1$ & 45 & 3.50 & 3.19 & 295 & 1.20 & 1490 & 8.7 \\
\hline $545-2$. & 46 & 2.93 & 2.66 & 322 & 1.20 & 1293 & 7.9 \\
\hline $545-3^{a} \ldots$ & 47 & 4.48 & 3.70 & 431 & 1.23 & 2116 & 12.7 \\
\hline $545-4 \quad \ldots$ & 48 & 2.76 & 2.51 & 297 & 1.19 & 1446 & 8.7 \\
\hline $857-1$ & 49 & 3.59 & 3.31 & 222 & 1.20 & 36566 & 3.4 \\
\hline $857-2$ & 50 & 4.10 & 3.75 & 265 & 1.15 & 36923 & 3.8 \\
\hline $857-3$ & 51 & 3.47 & 3.21 & 236 & 1.20 & 37037 & 3.5 \\
\hline $857-4^{a} \ldots \ldots \ldots$ & 52 & 3.64 & 3.00 & 622 & 1.09 & 50180 & 5.4 \\
\hline Dark1 $\ldots \ldots \ldots$ & 53 & 1.17 & 1.14 & 136 & 1.42 & 16496 & $\ldots$ \\
\hline Dark2 $\ldots \ldots \ldots$ & 54 & 1.39 & 1.35 & 148 & 1.40 & 19462 & $\ldots$ \\
\hline
\end{tabular}

Notes. NE $\Delta T$ is derived from $\mathrm{NEP}_{2} .{ }^{(a)}$ Bolometers suffering from random telegraphic signals (RTS).

values well above the noise (e.g., ten times the rms); 2) two, three, or more distinct levels can be observed for a given bolometer; and 3) the RTS is intermittent. For long periods of time, the RTS can be unnoticeable, especially for the $857-4$ bolometer.
Finally, we have observed examples of discrete jumps in the white noise level of several other bolometers at a rate of just a few every year. This non-stationary behavior appears unrelated to the RTS. 

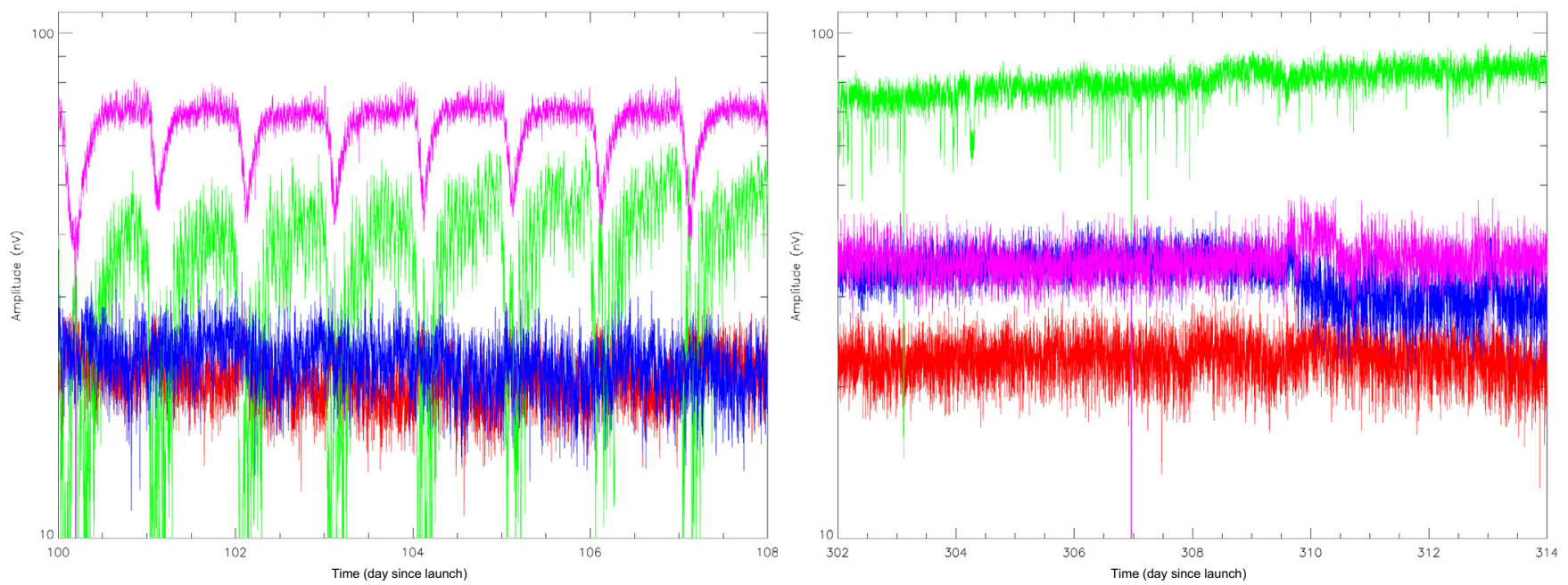

Fig. 16. Amplitudes vs. time of the four main lines produced by the $4 \mathrm{~K}$ cooler on the test resistor signal. Time units are Operation Days (OD), i.e. number of days since launch. (Left) Period when the transponder was switched on once per day for the 3-h Daily Tele-Communication Period. (Right) Later in the mission, when the transponder was always kept on.

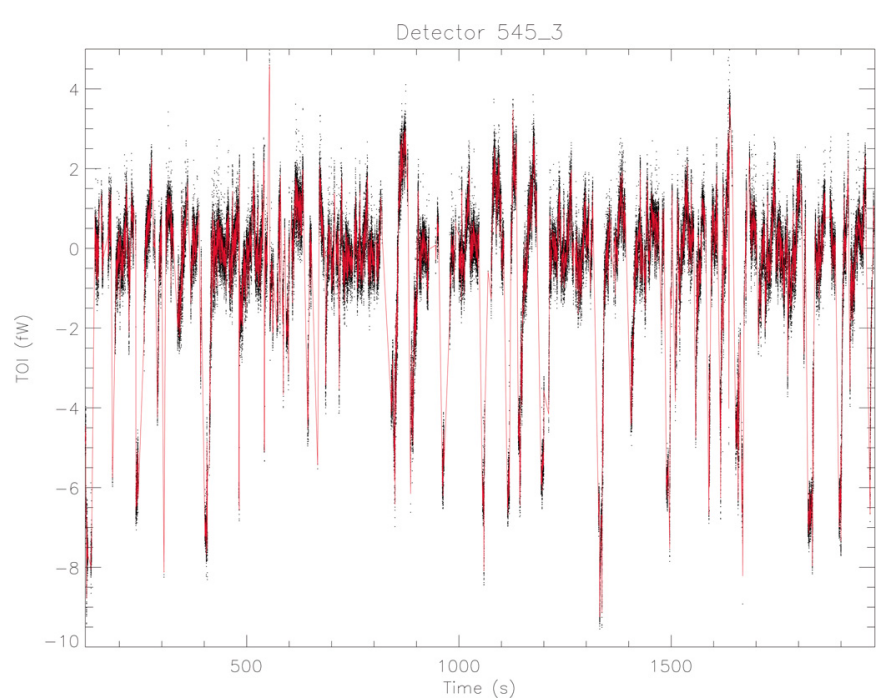

Fig. 17. Random telegraphic signal in the noise timeline (fW) of bolometer 545-3 plotted vs. time. RTS is here a two-level signal with random occurrences of flipping. Full sampling is in black dots. A smoothed version (by 41 samples) is plotted with a red line.

\subsection{Cosmic rays and their effects}

Energy is deposited by cosmic rays in various components of the HFI instrument. We observe these events in the TOIs of all detectors as a signature characterised by a very short rise time (less than $1.5 \mathrm{~ms}$ ) and an exponential decay. These events are called glitches. The other effect of the cosmic rays is a thermal input to the bolometer plate, which induces low frequency noise on the bolometers. The thermal effects are described in Planck HFI Core Team (2011b) and their impact on the long term stability are detailed in Sect. 7.

Cosmic rays consist of two main components at the L2 orbit of Planck: the Solar component and the Galactic component. The Solar component has low energy (a few keV) except during flares, when energies can reach to GeV. HFI is immune to the low energy component and no major flares have yet been

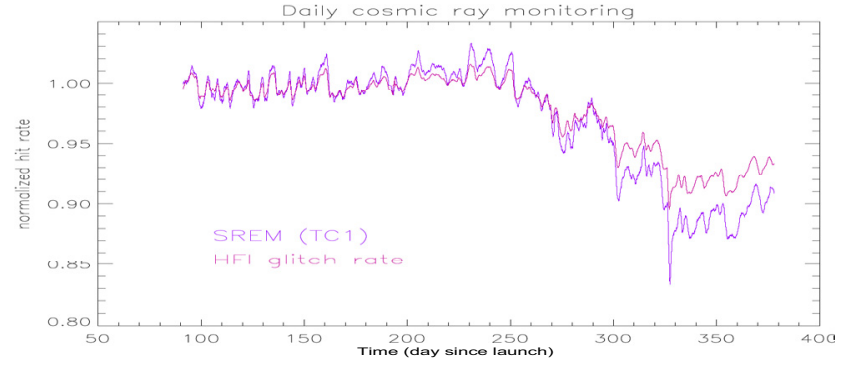

Fig. 18. SREM hit count and HFI bolometer average glitch rate evolution. SREM TC1 hit counts measure the protons with a deposited energy larger than $0.085 \mathrm{MeV}$.

recorded. The Galactic component (Shikaze et al. 2007), with a maximum between roughly $300 \mathrm{MeV}$ and $1 \mathrm{GeV}$, is modulated by Solar activity. The Planck mission began during the weakest solar activity for a century (McDonald et al. 2010). Hence, the Galactic cosmic ray component is expected to be at its highest level.

The evolution of the glitch rate (Fig. 18) follows closely the proton monitoring by the space radiation environment monitor (SREM), a piggy-back experiment mounted on the Planck spacecraft. This figure shows that cosmic rays are the main source of HFI glitches. The glitch rate has tended to decrease during the mission (since January 2010) due to the gradual increase of Solar activity.

The glitch rate can be understood as the sum of two interaction modes, corresponding to direct or indirect energy deposition on the bolometers. High energy cosmic rays can also interact with the bolometer plate and induce thermal effects and correlated glitches on the bolometers. These are dealt with in Sect. 7. The glitch characteristics further depend on the location of the energy deposition within the bolometer: the thermistor, the absorbing grid, or the bolometer housing. Typically thermistor hits are about $20 \mathrm{keV}$, whereas grid hits are about $2 \mathrm{keV}$. These events occur at a rate of a few per minute.

Cosmic ray particles can also deposit energy indirectly. All particles passing through matter produce a shower of secondary 


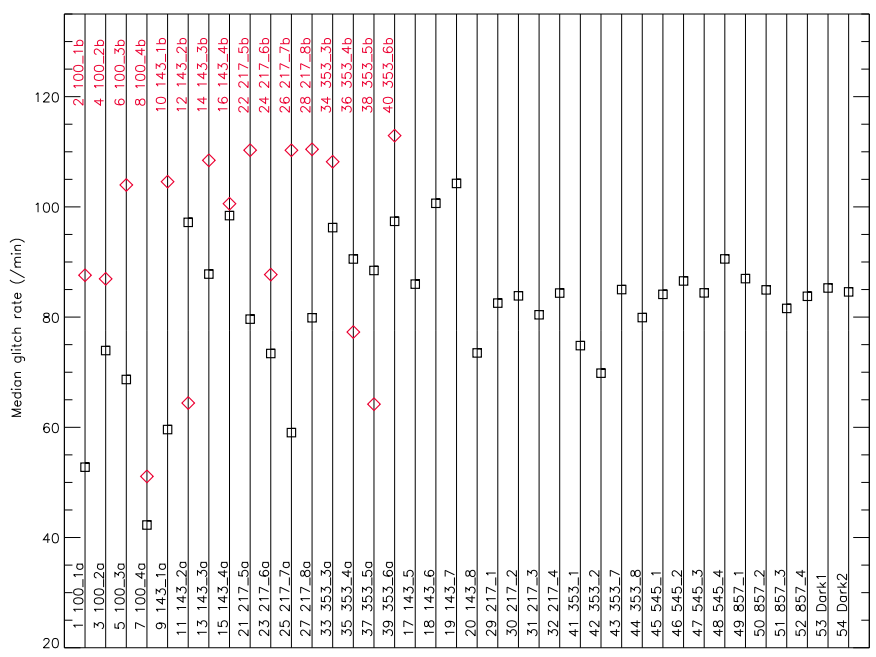

Fig. 19. Glitch rate of all HFI bolometers. An average over the first sky survey has been performed. The asymmetry between PSB bolometers sharing the same horn is an effect of detection threshold and asymmetric time constant properties between PSB $a$ and $b$.

electrons through ionization that are mostly absorbed in the nearby matter. However, interactions occurring within microns of the internal surface of the bolometer box produce a shower of free secondary particles. A fraction of the energy in these surface showers is absorbed by the thermistor and the grid of the bolometer. This explains the large coincidence rate of gliches between PSB bolometers $a$ and $b$ sharing the same mounting structure. The energy of those glitches follows a power law distribution spanning the whole range from the detection threshold to the saturation level. This spectrum is expected for the delta and secondary electrons produced via the ionization process. The total rate of these events is typically one per second, and thus dominates the total counts shown in Fig. 19.

The bolometer plate thermometers have a large cosmic particle hit rate (Planck Collaboration 2011b) because of their relatively large cross-section compared to that of the bolometers. This appears to be the dominant source of the low frequency excess noise present below about $100 \mathrm{mHz}$. The cosmic ray flux limits the sensitivity of the thermometry, precluding their use as a template for fine thermal fluctuations of the bolometer plate. Instead, the data processing pipeline (Planck HFI Core Team 2011b) uses blind bolometers located on the bolometer plate. A more detailed description of the effect of cosmic rays on HFI detectors is postponed to a dedicated paper, and glitch handling in the data processing is described by Planck HFI Core Team (2011b).

\section{Instrument stability}

The radiative power incident on each bolometer is the sum of the flux from the sky and of the thermal emission of all optical elements "seen" from the detector: filters, horns, telescope reflectors, shields and mechanical parts visible in the side-lobes. In addition, fluctuations of the heat sink temperature (the bolometer plate) appear as an optical signal. Any change in the parameters (temperature, emissivity, geometrical coefficient) driving these sources may be visible in the bolometer signal as a "DC level", i.e. a stable or very slowly varying (on the timescale of several days) component of the signal.

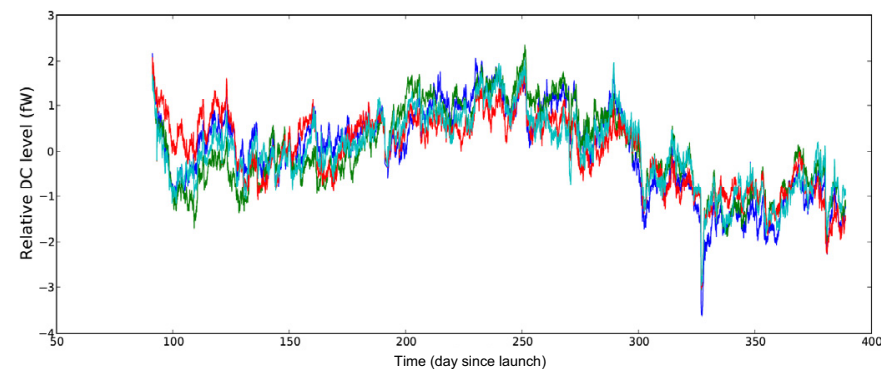

Fig. 20. The drift in the DC level of the $217 \mathrm{GHz}$ SWB bolometers, in femtoWatts of equivalent power in the detector, for the first year of operation.

Monitoring the "DC level" supposes that one is able to distinguish the instrumental effects from the sky signal. This is done in the map-making process (Planck HFI Core Team 2011b) by using the redundancy of the scanning strategy. Figure 20 shows for the $217 \mathrm{GHz}$ bolometers the history of the DC level during nearly one year. All follow a pattern similar to that of the cosmic ray activity measured by the SREM (see Sect. 6 and Fig. 18), which indicates that cosmic rays are the primary origin of the measured signal. One can check on families of bolometers with significantly different thermal conductance, $G$, that this signal is directly related to temperature variations of the bolometer plate and not to external optical sources. The similarity of Figs. 20 and 18, also shows that the effect of gain variations and of DC level drifts of the readout electronics is small with respect to other sources of signal drifts.

It should be noted that the "DC level" variation of the $217 \mathrm{GHz}$ bolometers is equivalent to an optical power of a couple of femtowatts, while the total background power on these bolometers is of order $1 \mathrm{pW}$. This fluctuation is mainly due to the energy deposited by cosmic rays on the bolometer plate, which means that the "equivalent power" of the other sources of temperature fluctuation and of optical background fluctuations are no more than a fraction of a femtowatt, i.e., less than one part per thousand of the background.

The change of gain induced by the DC level variations can be estimated from the non-linearity measurements (see Sect. 3.3.2). In the case considered in Fig. 20, the relative gain change is of the order of a few $\times 10^{-4}$.

During the CPV phase, the readout electronics were balanced, i.e., the offset parameter was tuned to get a signal near to zero. During the first year of operation, and for all bolometers, deviations from this point remained small with respect to the total range of acceptable values. As a consequence, no retuning of the readout electronics was needed during this period and it is expected to be the same up to the end of the mission.

\section{Main performance parameters}

The primary difference between the in-flight and pre-launch performance of the HFI derives from the relatively high rate of cosmic rays in the $L_{2}$ environment. At the energies of interest, the low level of solar activity results in an elevated cosmic ray flux. The glitches that result from cosmic ray events must be identified and removed from the time ordered information prior to processing the data into maps. The TOI processing also removes a significant fraction of the common mode component that appears in the bolometer TOIs at low frequencies. A residual lowfrequency component is largely removed during the map-making process (Planck HFI Core Team 2011b). 


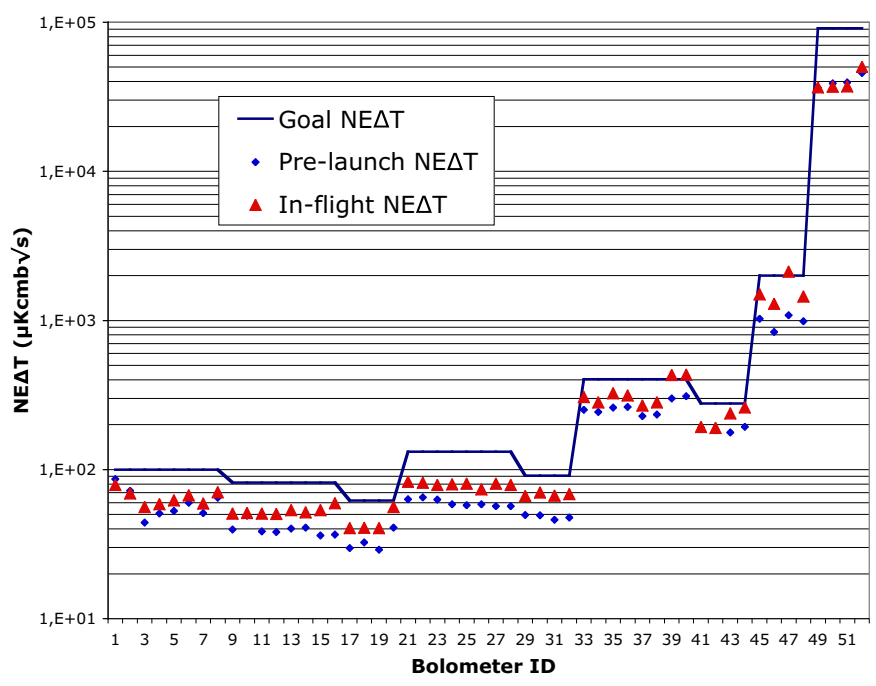

Fig. 21. Noise Equivalent Delta Temperature measured on the ground and in-flight with slightly different tools.

Table 6 summarizes the noise properties of the processed TOI (Planck HFI Core Team 2011b), by the following parameters:

- A white noise model. $\mathrm{NEP}_{1}$ is the average of the Noise Equivalent Power spectrum in the $0.6-2.5 \mathrm{~Hz}$ range.

- A model with a white noise $\mathrm{NEP}_{2}$ plus a low frequency component: $\mathrm{NEP}=\mathrm{NEP}_{2}\left[1+\left(f_{\mathrm{knee}} / f\right)^{\alpha}\right]$.

- The sensitivity $\mathrm{NE} \Delta T_{\mathrm{CMB}}$ to temperature differences of the CMB.

- The sensitivity NE $\Delta T_{\mathrm{RJ}}$ to temperature differences for sources observed in the Rayleigh-Jeans regime.

Figure 21 compares the goal, pre-launch and in-flight NEATs. The average in-flight NE $\Delta$ Ts are $27 \%$ higher than the pre-launch NE$\Delta T$ s. Differences between the pre-launch and in-flight values are due partly to differences in processing, but mostly to residual contamination from cosmic rays that is not completely removed in the current TOI processing.

The sensitivity goals are taken from Table 1 of Lamarre et al. (2010), and are consistent with Table 1.3 of Planck Collaboration (2005) corrected for the use of PSBs at $100 \mathrm{GHz}$. Note that Fig. 21 supersedes Fig. 11 of Lamarre et al. (2010) in which requirements and goals were improperly plotted. The in-flight noise levels estimated from $\mathrm{NEP}_{1}$ exceed the goals, which are defined by a total noise level equal to twice the expected contribution of photon noise. The average measured $\mathrm{NEP}_{1}$ is typically $70 \%$ of the initial goal. The improvement in the NEP over the design goals is primarily the result of having reduced the photon background through careful design of the optical system.

\section{Conclusions}

We report on the in-flight performance of the High Frequency Instrument on board the Planck satellite. These results are derived from the data obtained during a dedicated period of diagnostic testing prior to the initiation of the scientific survey, as well as an analysis of the survey data that form the basis of the early release scientific products.

With the exception of a single anomaly in the operation of the ${ }^{4} \mathrm{He}-\mathrm{JT}$ cooler, the HFI has operated nominally since launch. The settings of the readout electronics determined during prelaunch testing were found to be very near the optimal value in flight and were applied without any modification. A random telegraphic signal is observed in the same three channels that exhibited this behaviour during the final pre-launch testing. These channels are currently excluded from the scientific analysis. The instrument operation has been extremely stable during the first year of operation, requiring no adjustment of the readout electronics.

The optical design, and the alignment of the optical assembly, relied on both theoretical analysis and testing at the subsystem level. The beams of the 545 and $857 \mathrm{GHz}$ channels, which employ multimoded corrugated horns and waveguide, could not be measured on the ground. The actual beam widths of these channels measured on planets are in general smaller than the design goals and estimated values. The optical properties of the single mode channels are in excellent agreement with the design expectations.

A higher than expected cosmic ray flux, related to the level of Solar activity, results in a manageable loss of signal and degradation of thermal stability. Discrete cosmic ray events result in glitches in the scientific signal that are flagged and removed by an algorithm making use of the signal redundancy in the timeline. In addition to these single events, the cosmic ray flux contributes a significant thermal load on the sub-kelvin stage. Variations in this flux produce low-frequency fluctuations in the bolometer plate that induce a common mode component to the scientific signal and the focal plane thermometry. Although a component correlated with the dark bolometer outputs is removed during the TOI processing, a residual low frequency contribution is largely removed at the map-making stage. With the exception of the three detectors affected by telegraph noise, the sensitivity measured above $0.6 \mathrm{~Hz}$ exceeds the design goals of all HFI channels. After the removal of the residual low frequency noise, the final sensitivity of the frequency maps exceeds the mission requirements, and approaches the goals, as described in the companion paper Planck HFI Core Team (2011b).

Acknowledgements. The Planck HFI instrument (http://hfi.planck.fr/) was designed and built by an international consortium of laboratories, universities and institutes, with important contributions from the industry, under the leadership of the PI institute, IAS at Orsay, France. It was funded in particular by CNES, CNRS, NASA, STFC and ASI. The authors extend their gratitude to the numerous engineers and scientists, who have contributed to the design, development, construction or evaluation of the HFI instrument. A description of the Planck Collaboration and a list of its members, indicating which technical or scientific activities they have been involved in, can be found at http://www.rssd.esa.int/ index .php?project=PLANCK \\&page=Planck_Collaboration. The Planck Collaboration acknowledges financial support from: ESA; CNES and CNRS/INSU-IN2P3-INP (France); ASI, CNR, and INAF (Italy); NASA and DoE (USA); STFC and UKSA (UK); CSIC and MICINN (Spain); Tekes and AoF (Finland); DLR and MPG (Germany); CSA (Canada); DTU Space (Denmark); Swiss Funding Agency (Switzerland); Norwegian Funding Agency (Norway); and FCT/MCTES (Portugal).

\section{References}

Ade, P. A. R., Savini, G., Sudiwala, R., et al. 2010, A\&A, 520, A11

Benoît, A., Sirbi, A., Bradshaw, T., et al. 1997, in Sixth European Symposium on Space Environmental Control Systems, ed. T.-D. Guyenne, ESA SP, 400, 497

Benoît, A., Ade, P. A. R., Amblard, A., et al. 2003a, A\&A, 399, L25

Benoît, A., Ade, P. A. R., Amblard, A., et al. 2003b, A\&A, 399, L19

Bersanelli, M., Mandolesi, N., Butler, R. C., et al. 2010, A\&A, 520, A4

Bhandari, P., Bowman, R. C., Chave, R. G., et al. 2000, Astrophys. Lett. Commun., 37, 227

Bhandari, P., Prina, M., Bowman, R. C., et al. 2004, Cryogenics, 44, 395 
Bock, J. J., Chen, D., Mauskopf, P. D., \& Lange, A. E. 1995, Space Sci. Rev., 74, 229

Bradshaw, T. W., \& Orlowska, A. H. 1997, in Sixth European Symposium on Space Environmental Control Systems, ed. T.-D. Guyenne, ESA SP, 400, 465

Catalano, A. 2008, Ph.D. Thesis, École doctorale Astronomie et Astrophysique d'île de France

Catalano, A., Coulais, A., \& Lamarre, J.-M. 2010, Appl. Opt., 49, 5938

Church, S. E., Philhour, B. J., Lange, A. E., et al. 1996, in Submillimetre and Far-Infrared Space Instrumentation, ed. E. J. Rolfe, \& G. Pilbratt, ESA SP, 388, 77

Crill, B. P., Ade, P. A. R., Artusa, D. R., et al. 2003, ApJS, 148, 527

Gaertner, S., Benoît, A., Lamarre, J.-M., et al. 1997, A\&AS, 126, 151

Górski, K. M., Hivon, E., Banday, A. J., et al. 2005, ApJ, 622, 759

Holmes, W. A., Bock, J. J., Crill, B. P., et al. 2008, Appl. Opt., 47, 5996

Huffenberger, K. M., Crill, B. P., Lange, A. E., Górski, K. M., \& Lawrence, C. R. 2010, A\&A, 510, A58

Jensen, F., et al. 2010, Proc. Fourth European Conference on Antennas and Propagation (EUCAP)

Jones, W. C., Bhatia, R., Bock, J. J., \& Lange, A. E. 2003, in SPIE Conf. Ser. 4855, ed. T. G. Phillips, \& J. Zmuidzinas, 227

Jones, W. C., Ade, P. A. R., Bock, J. J., et al. 2006, ApJ, 647, 823

Lamarre, J.-M., Puget, J.-L., Ade, P. A. R., et al. 2010, A\&A, 520, A9

Leahy, J. P., Bersanelli, M., D’Arcangelo, O., et al. 2010, A\&A, 520, A8

Maffei, B., Noviello, F., Murphy, J. A., et al. 2010, A\&A, 520, A12

Mandolesi, N., Bersanelli, M., Butler, R. C., et al. 2010, A\&A, 520, A3

McDonald, F. B., Webber, W. R., \& Reames, D. V. 2010, Geophys. Res. Lett., 37,18101

Mennella, A., Bersanelli, M., Butler, R. C., et al. 2011, A\&A, 536, A3

Montroy, T. E., Ade, P. A. R., Bock, J. J., et al. 2006, ApJ, 647, 813

Murphy, J. A., Colgan, R., O'Sullivan, C., Maffei, B., \& Ade, P. A. R. 2001, Infrared Physics and Technology, 42, 515

Murphy, J. A., Colgan, R., Gleeson, E., et al. 2002, in Experimental Cosmology at Millimetre Wavelengths, ed. M. de Petris, \& M. Gervasi, Am. Inst. Phys. Conf. Ser., 616, 282

Murphy, J. A., Peacocke, T., Maffei, B., et al. 2010, J. Instrum., 5, 4001

Pajot, F., Ade, P. A. R., Beney, J., et al. 2010, A\&A, 520, A10

Piacentini, F., Ade, P. A. R., Bock, J. J., et al. 2006, ApJ, 647, 833

Planck Collaboration 2005, ESA publication ESA-SCI(2005)/01

Planck Collaboration 2011a, A\&A, 536, A1

Planck Collaboration 2011b, A\&A, 536, A2

Planck Collaboration 2011c, A\&A, 536, A7

Planck Collaboration 2011d, A\&A, 536, A8

Planck Collaboration 2011e, A\&A, 536, A9

Planck Collaboration 2011f, A\&A, 536, A10

Planck Collaboration 2011g, A\&A, 536, A11

Planck Collaboration 2011h, A\&A, 536, A12

Planck Collaboration 2011i, A\&A, 536, A13

Planck Collaboration 2011j, A\&A, 536, A14

Planck Collaboration 2011k, A\&A, 536, A15

Planck Collaboration 20111, A\&A, 536, A16

Planck Collaboration 2011m, A\&A, 536, A17

Planck Collaboration 2011n, A\&A, 536, A18

Planck Collaboration 2011o, A\&A, 536, A19

Planck Collaboration 2011p, A\&A, 536, A20

Planck Collaboration 2011q, A\&A, 536, A21

Planck Collaboration 2011r, A\&A, 536, A22

Planck Collaboration 2011s, A\&A, 536, A23

Planck Collaboration 2011t, A\&A, 536, A24

Planck Collaboration 2011u, A\&A, 536, A25

Planck Collaboration 2011v, The Explanatory Supplement to the Planck Early

Release Compact Source Catalogue (ESA)

Planck Collaboration 2011w, A\&A, 536, A26

Planck HFI Core Team 2011a, A\&A, 536, A4

Planck HFI Core Team 2011b, A\&A, 536, A6

Pratt, W. K. 1978, Digital image processing, ed. W. K. Pratt

Rieke, F. M., Lange, A. E., Beeman, J. W., \& Haller, E. E. 1989, IEEE Transactions on Nuclear Science, 36, 946

Rosset, C., Tristram, M., Ponthieu, N., et al. 2010, A\&A, 520, A13

Ruze, J. 1966, Proceedings of the IEEE, 54, 633

Shikaze, Y., Haino, S., Abe, K., et al. 2007, Astropart. Phys., 28, 154

Sudiwala, R. V., Maffei, B., Griffin, M. J., et al. 2000, Nuclear Instruments and

Methods in Physics Research A, 444, 408

Swinyard, B. M., Hartogh, P., Sidher, S., et al. 2010, A\&A, 518, L151

Tauber, J. A., Mandolesi, N., Puget, J.-L., et al. 2010a, A\&A, 520, A1
Tauber, J. A., Norgaard-Nielsen, H. U., Ade, P. A. R., et al. 2010b, A\&A, 520, A2

Zacchei, A., Maino, D., Baccigalupi, C., et al. 2011, A\&A, 536, A5

1 Astroparticule et Cosmologie, CNRS (UMR 7164), Université Denis Diderot Paris 7, Bâtiment Condorcet, 10 rue A. Domon et Léonie Duquet, Paris, France

2 Astrophysics Group, Cavendish Laboratory, University of Cambridge, J J Thomson Avenue, Cambridge CB3 OHE, UK

3 Atacama Large Millimeter/submillimeter Array, ALMA Santiago Central Offices, Alonso de Cordova 3107, Vitacura, Casilla 763 0355, Santiago, Chile

4 CITA, University of Toronto, 60 St. George St., Toronto, ON M5S 3H8, Canada

5 CNES, 18 avenue Édouard Belin, 31401 Toulouse Cedex 9, France

6 CNRS, IRAP, 9 Av. colonel Roche, BP 44346, 31028 Toulouse Cedex 4, France

7 California Institute of Technology, Pasadena, California, USA

8 DAMTP, University of Cambridge, Centre for Mathematical Sciences, Wilberforce Road, Cambridge CB3 0WA, UK

9 DSM/Irfu/SPP, CEA-Saclay, 91191 Gif-sur-Yvette Cedex, France

10 DTU Space, National Space Institute, Juliane Mariesvej 30, Copenhagen, Denmark

11 Department of Astronomy and Astrophysics, University of Toronto, 50 Saint George Street, Toronto, Ontario, Canada

12 Department of Physics, Princeton University, Princeton, New Jersey, USA

13 Department of Physics, University of California, One Shields Avenue, Davis, California, USA

14 Department of Physics, University of Illinois at Urbana-Champaign, 1110 West Green Street, Urbana, Illinois, USA

15 Dipartimento di Fisica, Università La Sapienza, P. le A. Moro 2, Roma, Italy

16 Dipartimento di Fisica, Università degli Studi di Milano, via Celoria, 16, Milano, Italy

17 European Southern Observatory, ESO Vitacura, Alonso de Cordova 3107, Vitacura, Casilla 19001, Santiago, Chile

18 European Space Agency, ESTEC, Keplerlaan 1, 2201 AZ Noordwijk, The Netherlands

19 INAF - Osservatorio Astronomico di Trieste, via G.B. Tiepolo 11, Trieste, Italy

20 INAF/IASF Bologna, via Gobetti 101, Bologna, Italy

21 INAF/IASF Milano, via E. Bassini 15, Milano, Italy

22 INSU, Institut des sciences de l'univers, CNRS, 3 rue Michel-Ange, 75794 Paris Cedex 16, France

23 IPAG: Institut de Planétologie et d'Astrophysique de Grenoble, Université Joseph Fourier, Grenoble 1/CNRS-INSU, UMR 5274, 38041 Grenoble, France

24 Imperial College London, Astrophysics group, Blackett Laboratory, Prince Consort Road, London, SW7 2AZ, UK

25 Infrared Processing and Analysis Center, California Institute of Technology, Pasadena, CA 91125, USA

26 Institut Néel, CNRS, Université Joseph Fourier Grenoble I, 25 rue des Martyrs, Grenoble, France

27 Institut d'Astrophysique Spatiale, CNRS (UMR8617) Université Paris-Sud 11, Bâtiment 121, Orsay, France

28 Institut d'Astrophysique de Paris, CNRS UMR7095, Université Pierre \& Marie Curie, 98bis boulevard Arago, Paris, France

29 Institut de Ciències de l'Espai, CSIC/IEEC, Facultat de Ciències, Campus UAB, Torre C5 par-2, Bellaterra 08193, Spain

30 Institut de Radioastronomie Millimétrique (IRAM), Avenida Divina Pastora 7, Local 20, 18012 Granada, Spain

31 Institut de Radioastronomie Millimétrique (IRAM), Domaine Universitaire de Grenoble, 300 rue de la Piscine, 38406 Grenoble, France 
32 Institute of Astronomy, University of Cambridge, Madingley Road, Cambridge CB3 0HA, UK

33 Instituto de Astrofísica de Canarias, C/Vía Láctea s/n, La Laguna, Tenerife, Spain

34 Jet Propulsion Laboratory, California Institute of Technology, 4800 Oak Grove Drive, Pasadena, California, USA

35 Jodrell Bank Centre for Astrophysics, Alan Turing Building, School of Physics and Astronomy, The University of Manchester, Oxford Road, Manchester, M13 9PL, UK

36 Kavli Institute for Cosmology Cambridge, Madingley Road, Cambridge, CB3 OHA, UK

37 LERMA, CNRS, Observatoire de Paris, 61 avenue de l'Observatoire, Paris, France

${ }^{38}$ Laboratoire AIM, IRFU/Service d'Astrophysique - CEA/DSM CNRS - Université Paris Diderot, Bât. 709, CEA-Saclay, 91191 Gifsur-Yvette Cedex, France

39 Laboratoire Traitement et Communication de l'Information, CNRS (UMR 5141) and Télécom ParisTech, 46 rue Barrault, 75634 Paris Cedex 13, France

40 Laboratoire d'Astrophysique de Marseille, 38 rue Frédéric JoliotCurie, 13388, Marseille Cedex 13, France

41 Laboratoire de Physique Subatomique et de Cosmologie, CNRS/IN2P3, Université Joseph Fourier Grenoble I, Institut National Polytechnique de Grenoble, 53 rue des Martyrs, 38026 Grenoble Cedex, France

${ }^{42}$ Laboratoire de l'Accélérateur Linéaire, Université Paris-Sud 11, CNRS/IN2P3, Orsay, France

43 Lawrence Berkeley National Laboratory, Berkeley, California, USA

44 Max-Planck-Institut für Astrophysik, Karl-Schwarzschild-Str. 1, 85741 Garching, Germany
45 National University of Ireland, Department of Experimental Physics, Maynooth, Co. Kildare, Ireland

46 Observational Cosmology, Mail Stop 367-17, California Institute of Technology, Pasadena, CA, 91125, USA

47 Optical Science Laboratory, University College London, Gower Street, London, UK

48 Rutherford Appleton Laboratory, Chilton, Didcot, UK

49 SUPA, Institute for Astronomy, University of Edinburgh, Royal Observatory, Blackford Hill, Edinburgh EH9 3HJ, UK

50 School of Physics and Astronomy, Cardiff University, Queens Buildings, The Parade, Cardiff, CF24 3AA, UK

51 Space Research Institute (IKI), Russian Academy of Sciences, Profsoyuznaya Str, 84/32, Moscow 117997, Russia

52 Space Sciences Laboratory, University of California, Berkeley, California, USA

53 Stanford University, Dept of Physics, Varian Physics Bldg, 382 via Pueblo Mall, Stanford, California, USA

54 Universität Heidelberg, Institut für Theoretische Astrophysik, Albert-Überle-Str. 2, 69120 Heidelberg, Germany

55 Université de Toulouse, UPS-OMP, IRAP, 31028 Toulouse Cedex 4, France

56 Universities Space Research Association, Stratospheric Observatory for Infrared Astronomy, MS 211-3, Moffett Field, CA 94035, USA

57 University of Granada, Departamento de Física Teórica y del Cosmos, Facultad de Ciencias, Granada, Spain

58 University of Miami, Knight Physics Building, 1320 Campo Sano Dr., Coral Gables, Florida, USA

59 Warsaw University Observatory, Aleje Ujazdowskie 4, 00-478 Warszawa, Poland 